\title{
Die technowissenschaftlichen Laboratorien der Frühen Neuzeit
}

\author{
Ursula Klein
}

\begin{abstract}
Technoscientific Laboratories in Early Modern Europe
In the early modern period laboratories existed, as a rule, only in alchemy and chemistry as well as in innovative arts and crafts which employed chemical operations, such as pharmacy, metallurgy and the making of fireworks. The paper presents a comparative analysis of the material culture of pharmaceutical and academic laboratories in eighteenth-century Germany. Based on this analysis, it is argued that until around 1800 laboratories were technoscientific sites of both learned inquiries into nature, technological investigations, and manufacture. This argument is supported by the local circumstances of the specific historical case described in the second part of the paper: the way in which the laboratory of the Royal Prussian Academy of Sciences was equipped with instruments, vessels and materials. The first director of this academic laboratory, the apothecary-chemist Andreas Sigismund Marggraf, as well as its third director, Martin Heinrich Klaproth, equipped the academic laboratory with the instruments, vessels and materials coming from their own pharmaceutical laboratories. There was thus a direct material transfer from an artisanal laboratory to an academic laboratory.
\end{abstract}

Keywords: early modern laboratories, laboratory of the Prussian Academy of Sciences, material culture, material transfer, technoscience

Schlüsselwörter: Laboratorien in der Frühen Neuzeit, Laboratorium der Preußischen Akademie der Wissenschaften, materielle Kultur, materieller Transfer, Technowissenschaften

Laboratorien gelten heute als Embleme wissenschaftlichen Experimentierens und als die privilegierten Orte wissenschaftlichen Forschens und Lehrens in vielen verschiedenen Disziplinen, von der experimentellen Physik, Chemie und Biologie bis hin zur Psychologie. Das war nicht immer so. Einer der berühmtesten Experimentatoren der Frühen Neuzeit, Robert Hooke (1635-1703), experimentierte nicht in einem Laboratorium (Cooper 2003, Shapin 1988, 1989, Simpson 1989). Als curator of experiments der Royal Society führte Hooke bei den wöchentlichen Zusammenkünften der Londoner Royal Society Experimente vor. Diese Demonstrationsexperimente bereitete er in seiner im Gresham College gelegenen Wohnung vor, in der er auch Instrumente herstellte. Die Zusammenkünfte der Royal Society fanden zuerst in einem Raum im Gresham College statt, der für den Professor für Geome- 
trie vorgesehenen war und den ein französischer Besucher als eine Art „Amphitheater" oder Vorlesungshalle beschrieb (Shapin 1988: 391). Als das Gebäude 1666 nach der großen Brandkatastrophe in London wieder hergestellt war, trafen sich die Mitglieder der Royal Society dagegen in einem eigens für sie eingerichteten Versammlungsraum. Hooke führte seine Experimente ausschließlich in diesem Raum vor, denn entgegen ihren ursprünglichen Plänen errichtete die Royal Society kein eigenes Laboratorium (Shapin 1988). Das war aber keineswegs ungewöhnlich, denn in der Frühen Neuzeit wurde an vielen verschiedenen Orten experimentiert, in öffentlichen Versammlungsräumen, Kaffeehäusern, privaten Salons, Stuben und Küchen, und nur in einem spezifischen Bereich, um den es im Folgenden geht, ausschließlich in speziell eingerichteten "Laboratorien".

Anders als Hooke verfügte ein zweiter berühmter britischer Experimentator des 17. Jahrhunderts, Robert Boyle (1627-1691), sehr wohl über Laboratorien, die er für seine chemischen Experimente benutzte (Pilcher 1938, Sargent 1995, Shapin 1988). Im Erdgeschoss des 1683 eröffneten Ashmolean Museum in Oxford befand sich ebenfalls ein chemisches Laboratorium, das als erstes zu Lehrzwecken eingerichtetes Laboratorium Englands gilt (Simcock 1984). Die kurz nach der Royal Society gegründete Pariser Académie Royale des Sciences verfügte schon früh über ein Laboratorium an ihrem Standort in der Königlichen Bibliothek in der Rue Vivienne (Meynell 1994, Stroup 1990). Auch dieses war ein chemisches Laboratorium, wie auch das zweite öffentliche für Naturforschung und Demonstrationsexperimente errichtete Laboratorium im Pariser Jardin Royal des Plantes. In Deutschland gab es ebenfalls bereits im 17. Jahrhundert Laboratorien für Lehr- und Forschungszwecke wie das an der Universität Marburg, wo Johannes Hartmann lehrte (Moran 1991), und das an der Universität Altdorf. Auch in diesen beiden Fällen handelte es sich um chemische Laboratorien. Im 16. Jahrhundert hatte die Ausbreitung der Alchemie an Fürstenhöfen zur Einrichtung zahlreicher alchemistischer Laboratorien geführt (Weyer 1992). So beauftragte 1577 die Gemahlin des Kurprinzen Joachim Friedrich den Alchemisten Leonhard Thurneysser, auf der Moritzburg in Halle ein alchemistisches Laboratorium einzurichten. Dieses dürfte Joachim Friedrich einige Jahre später veranlasst haben, im Zusammenhang mit der Einrichtung einer Hofapotheke im Berliner Schloss auch ein "geheim Laboratorium“ zu gründen (Hörmann 1898: 212). Die Liste der Beispiele ließe sich fortsetzen und beträchtlich erweitern für das 18. und frühe 19. Jahrhundert, wobei „Laboratorien“ fast immer speziell eingerichtete Räume für chemische Experimente und chemische Verfahren waren.

In den Lexika der Frühen Neuzeit, ${ }^{1}$ aber auch in anderen Literaturgattungen dieser Zeit, bezieht sich der Terminus „Laboratorium“ fast immer auf einen Raum oder ein Haus, in dem chemische Operationen durchgeführt wurden, in dem also Stoffe destilliert, geschmolzen, verbrannt, aufgelöst und 
präzipitiert wurden, um nur die wichtigsten Operationen zu nennen, die als "chemische“ galten. ${ }^{2}$ Der zweite potentielle Kandidat für frühneuzeitliche Laboratorien, die experimentelle Physik oder Experimentalphilosophie (experimental philosophy), errichtete dagegen "physikalische Kabinette" und "physikalische Theater“ (Wiesenfeldt 2002). Bei den „physikalischen Kabinetten" handelte es sich jedoch in der Regel um Räume, die dem Aufbewahren von Instrumenten dienten. Die Instrumente wurden in der Regel entliehen, um damit andernorts zu experimentieren. Die "physikalischen Theater“ waren dagegen in erster Linie Vorlesungsräume, in denen auch Modelle von Maschinen und experimentelle Effekte vorgeführt wurden. Sie standen in der Beobachtungs- und Lehrtradition der älteren anatomischen Theater und waren somit zwar Orte von Demonstrationsexperimenten, aber nur in Ausnahmefällen auch von explorierenden Experimenten (experimental trials), die nicht im unmittelbaren Zusammenhang von Lehr- und Demonstrationszwecken standen (Turner 1987: 190-202).

Bis Ende des 18. Jahrhunderts gab es Laboratorien im Sinne speziell ausgestatteter Räume des naturforschenden oder „wissenschaftlichen“ Experimentierens nahezu ausschließlich in der Chemie beziehungsweise Alchemie, und nur in der Chemie waren die Laboratorien auch die privilegierten Orte des Experimentierens. ${ }^{3}$ „Es ist (demnach) für einen jeden, welcher ein Chymist werden will, eine unumgängliche Sache, ein mit denen zur Ausübung dieser Wissenschaft nöthigsten Werkzeugen versehenes Laboratorium zu haben“, schrieb der französische Chemiker Pierre Joseph Macquer (17181784) in seinem berühmten chemischen Wörterbuch (Macquer 1766, 2: 3, meine Hervorhebung). Bis ins 19. Jahrhundert hinein war der Terminus „Laboratorium" in der Regel gleichbedeutend mit "chemisches Laboratorium". Dieser eng auf die Chemie beziehungsweise Alchemie bezogene Wortgebrauch von „Laboratorium“, der eine große Tragweite für die Historisierung unseres Verständnisses der Entstehung und Entwicklung der experimentellen Wissenschaften besitzt, ist bereits von mehreren Historikerinnen und Historikern hervorgehoben worden. ${ }^{5}$ Dagegen ist eine zweite historische Besonderheit dieses Wortgebrauchs außerhalb eines kleinen Kreises von Spezialisten für Alchemie und frühmoderne Chemie so gut wie unbeachtet geblieben: Als „Laboratorien“ galten in der Frühen Neuzeit nicht nur Orte des „wissenschaftlichen“ oder „akademischen“ Experimentierens, sondern auch besondere Typen handwerklicher Werkstätten. Im Folgenden diskutiere ich zuerst die Problematik der Interdependenz handwerklicher und „akademischer", das heißt vorwiegend der Naturforschung dienender Laboratorien der Frühen Neuzeit. „Akademische Laboratorien“ im hier verwendeten Sinn gab es im 18. Jahrhundert sowohl an Universitäten, Akademien, botanischen Gärten und Museen als auch in Form privater Laboratorien gelehrter Chemiker. ${ }^{6}$ Danach gehe ich konkreter auf Architektur und Ausstattung von Apothekerlaboratorien, die am weitesten verbreiteten handwerklich-ge- 
werblichen Laboratorien der Frühen Neuzeit, und akademische Laboratorien in der zweiten Hälfte des 18. Jahrhunderts ein. Dem folgt der zentrale Teil, in dem es um die Art und Weise der Ausstattung des Laboratoriums der Königlich Preußischen Akademie der Wissenschaften und um dessen enge Verflechtung mit Apothekerlaboratorien geht.

\section{Die Interdependenz handwerklicher und akademischer Laboratorien}

Naturbeobachtung und Naturforschung waren in der Frühen Neuzeit häufig verknüpft mit handwerklicher Innovation und Kommerz. ${ }^{7}$ Auch das lateinische Wort „laborare“ (arbeiten), von dem Laboratorium abgeleitet ist, verweist in diese Richtung. Ein „Laboratorium“ war ein „Arbeits-“ oder „Werkhaus", und beide Termini wurden in deutschen Texten des 18. Jahrhunderts häufig synonym verwendet. So übersetzt die Oekonomische Encyclopädie (1773-1858) des Kameralisten Johann Georg Krünitz „Laboratorium“ wörtlich mit „Arbeits- oder Werks-Haus“ und führt als instruktive Beispiele an erster Stelle die Laboratorien in der Chemie und Pharmazie an. „Laboratorium“, so heißt es, nennt man in der „Chemie und Pharmacie [...] den Ort, wo zu der chemischen Arbeit, zum Destillieren, Schmelzen, und zu andern dergleichen Dingen nöthige Gelegenheit zugerichtet, und die gehörigen Gefäße, an Brenn-Kolben, Gläsern, Retorten, Tiegeln, u.s.w. angeschaffet zu befinden sind.“ (Krünitz 1773-1858, 58: 47) Es folgen die Laboratorien der „BüchsenMeisterey oder Feuerwerker-Kunst" und die Werkstätten, in denen Öfen für die Schrift-Gießerei hergestellt werden (ebd., 58: 48, 18: 421). Das Universal Lexikon von Johann Heinrich Zedler übersetzt den Terminus ebenfalls mit „Arbeit- oder Werck-Haus“ und hebt den folgenden Typus von Laboratorium hervor: "Laboratorium, heisset in einem besonderen Verstande in der Artillerie derjenige Ort, an welchem das Pulver, und andere zum Feuerwerck gehörige Materien, erarbeitet und die Ernst- und Lust-Feuerwercke verfertiget werden." (Zedler 1732-1750, 16: 30) Erinnert sei in diesem Zusammenhang an das zum Berliner Zeughaus gehörende "Laboratorium“ und an das im 17. Jahrhundert gegründete "Royal Laboratory“ in Woolwich, im dem die königliche Schießpulverherstellung Englands kontrolliert wurde (Mauskopf 2006). Darüber hinaus wurden „Laboratorien“ auch in Münzen, Schmelzhütten, metallurgischen Probierwerkstätten und Destillierhäusern errichtet sowie in staatlichen Manufakturen wie zum Beispiel den Pariser Gobelins, wo sich im 18. Jahrhundert ein „Laboratorium“ zur chemischen Qualitätskontrolle von Farbstoffen befand (Nieto-Galan 2001).

Die am weitesten verbreiteten Laboratorien der Frühen Neuzeit waren jedoch die in den einzelnen Apotheken errichteten Apothekerlaboratorien. Diese dienten vorwiegend der gewerblichen Herstellung chemischer Me- 
dikamente, waren jedoch im 18. Jahrhundert zugleich auch wichtige Orte der Naturbeobachtung und Naturforschung (Dilg 2000, Klein 2007a, b, in Vorbereitung, Schneider 1972). Insgesamt handelte es sich bei den als „Laboratorien" bezeichneten gewerblichen Produktionsstätten der Frühen Neuzeit um innovative Typen von Werkstätten, in denen chemische Techniken angewandt und Expertenwissen über Natur und Technik weiterentwickelt wurden. Im Vergleich zu den handwerklich-gewerblichen Laboratorien lag der Schwerpunkt der frühneuzeitlichen akademischen Laboratorien auf $\mathrm{Na-}$ turforschung. Daneben beschäftigten sich die akademischen Chemiker des 18. Jahrhunderts aber auch mit pharmazeutischen und anderen technologischen Innovationen, und bis Mitte des 18. Jahrhunderts stellten sie in ihren Laboratorien auch direkt zum Verkauf vorgesehene chemische Medikamente her (Klein/Lefèvre 2007, Smith 2006).

Die weite Verwendung des Wortes „Laboratorium“ in der Frühen Neuzeit sowohl für besondere Typen von Werkstätten als auch für Institutionen des naturforschenden oder „wissenschaftlichen“ Experimentierens, so meine These, ist nicht nur als sprachliche Besonderheit zu bewerten. Vielmehr ist sie auch Ausdruck faktischer Interdependenz chemischer Naturforschung in akademischen Institutionen (oder in privaten Laboratorien gelehrter Alchemisten und Chemiker) und gewerblich-chemischer Praxis. Diese Interdependenz ging mit großen Übereinstimmungen in Architektur und Ausstattung der Laboratorien sowie in den dort angewandten Beobachtungstechniken und Verfahrensweisen einher. Die Übereinstimmungen in der materiellen Kultur und Praxis handwerklicher und akademischer Laboratorien waren eine notwendige, allerdings keine hinreichende Bedingung für nahtlose Übergänge vom vorrangigen Ziel der gewerblichen Produktion oder technologischen Innovation zu dem der Naturbeobachtung und -forschung oder umgekehrt. ${ }^{8}$ Sie machten es zum Beispiel ambitionierten Apothekern möglich, im Verlauf des Herstellungsprozesses chemischer Medikamente im Apothekerlaboratorium - oder auch neben und zusätzlich zu den Herstellungsprozessen - auffällige Phänomene zu beobachten und zu notieren, die sowohl für die Innovation in der Medikamentenherstellung als auch für das Verständnis der „Natur“ chemischer Stoffe und ihrer Reaktionen relevant sein konnten (Klein 2007b, in Vorbereitung). Umgekehrt ermöglichten sie gelehrten Alchemisten und akademischen Chemikern im 18. Jahrhundert, chemische Analysen der Zusammensetzung von Stoffen und Experimente zum Studium ihrer chemischen Reaktionen auch in technologischer Hinsicht auszuwerten und die Produkte dieser Experimente als Prototypen von Medikamenten oder anderen kommerziellen Gütern zu vermarkten (Klein/ Lefèvre 2007, Smith 1994, 2006). Diese Art „wissenschaftlich-technologischer" Zweigleisigkeit war nicht nur der Übereinstimmung von Instrumenten, Gefäßen und Materialien in akademischen und handwerklich-gewerblichen Laboratorien geschuldet, sondern auch den Übereinstimmungen der 
Beoachtungs- und Verfahrenstechniken. Bei den "chemischen Analysen“ des 18. Jahrhunderts handelte es sich nämlich in der Regel um produktive Herstellungsverfahren, bei denen die unsichtbaren Komponenten der Stoffe als materielle Stoffe isoliert wurden. Diese analytischen („wissenschaftlichen“) Verfahren stimmten oft selbst in der Größenordung mit den Methoden der gewerblichen Herstellung chemischer Medikamente, der gewerblichen chemischen Qualitätskontrolle von Farbstoffen und Schießpulver oder dem metallurgischen Probieren des Metallgehalts von Erzen, Münzen und Goldschmiedearbeiten überein. Dasselbe gilt für die meisten chemischen Reaktionen, die in der Frühen Neuzeit zu experimentellen Untersuchungsgegenständen gemacht wurden. Auch bei diesen Experimenten handelte es sich um materielle Herstellungsverfahren, deren unmittelbare Ergebnisse weder Daten noch andere Inskriptionen, sondern stoffliche Reaktionsprodukte waren.

Wegen dieser Verknüpfung von Analyse und Stoffherstellung und des häufigen Übergangs der Handlungsziele von gewerblicher Herstellung oder Versuchen technologischer Innovation zu Naturbeobachtung und intensiver Naturforschung (und umgekehrt), der in Laboratorien institutionalisiert war und damit eine dauerhafte und sozial akzeptierte Form besaß, bildeten die Chemie des 18. Jahrhunderts (wie auch die Alchemie in den Jahrhunderten zuvor) und die mit ihr assoziierten Gewerbe eine Einheit, die als „technoscience avant la lettre" charakterisiert werden kann (Klein 2005). Die Laboratorien der Frühen Neuzeit, einschließlich derjenigen des 18. Jahrhunderts, waren hybride Institutionen, in denen Naturbeobachtungen und Experiment mit handwerklich-gewerblicher Produktion und Innovation auf vielfältige Weise verwoben waren. Die Gemeinsamkeiten in der materiellen Kultur der frühneuzeitlichen Laboratorien, die im vorliegenden Aufsatz untersucht werden, waren die wichtigste Voraussetzung für derartige praktisch-institutionelle Mischbildungen und die nahtlosen Übergänge von gewerblicher Produktion zu Naturforschung und umgekehrt.

Die institutionellen Räume des Wissens sind in der neueren Wissenschaftsgeschichte als historiographische Einheiten verstanden worden, die das Studium der Praxis der Wissenschaften ermöglichen sollen (Livingstone 2003, Shapin 1988, Berichte zur Wissenschaftsgeschichte 23, 2000). Laboratorien sind Orte des Experimentierens, deren Geschichte jedoch noch weitgehend im Dunkeln liegt. Die Frage, welche Konsequenzen aus der Tatsache zu ziehen sind, dass es in der Experimentalphilosophie beziehungsweise experimentellen Physik vor circa 1800 nur in Ausnahmefällen Laboratorien, das heißt speziell ausgestattete und systematisch genutzte Räume des Experimentierens, gab, muss an dieser Stelle offen bleiben. ${ }^{9}$ Die Tatsache, dass in der Experimentalphilosophie Laboratorien äußerst selten, in der frühneuzeitlichen Chemie dagegen die Regel waren, ist darüber hinaus Ausgangspunkt für eine weitere Facette meines Arguments, die im vorliegen- 
den Aufsatz jedoch ebenfalls nicht vertieft diskutiert wird: In der Frühen Neuzeit entwickelte sich eine von der Experimentalphilosophie verschiedene Tradition des Experimentierens, die eingebettet war in avancierte Bereiche handwerklich-gewerblicher Produktion wie das Apothekergewerbe, die metallurgische Probierkunst, das Artilleriewesen, die Farbenherstellung, die Keramik- und Porzellanherstellung, die Destillationskunst, Versuche zur Intensivierung der Landwirtschaft und andere gewerbliche Praktiken. Der Ort dieser zweiten Tradition war das Laboratorium, das akademische und das handwerklich-gewerbliche, und die wissenschaftliche Disziplin war die Chemie, in der die verschiedenen Laboratoriumspraxen gebündelt und gelehrt sowie mit naturphilosophischen Traditionen in Berührung gebracht wurden. In der Chemie des 17. und 18. Jahrhunderts flossen verschiedene Stile des Experimentierens zusammen: Experimentalphilosophie, Experimentalgeschichte und „technologische“ Innovationen, die der Verbesserung der handwerklichen Künste und der Landwirtschaft dienten (Klein/Lefèvre 2007: $21-58) .{ }^{10}$

Die Betonung der historischen Tatsache, dass die „Laboratorien" der Frühen Neuzeit, einschließlich derjenigen des 18. Jahrhunderts, nicht nur Orte „wissenschaftlichen“ Experimentierens, sondern auch technologische Innovations- und handwerkliche Produktionsstätten waren, mag wie eine Provokation wirken. Auch in der Chemiegeschichte selbst gibt es keine Übereinstimmung hinsichtlich der historischen und epistemologischen Relevanz dieser Tatsache. Gelegentlich wurde sie mit wenigen Sätzen konstatiert, nicht selten wegargumentiert und häufig gänzlich ignoriert, vermutlich weil sie für irrelevant oder zumindest nicht für ein interessantes Problem gehalten wurde. ${ }^{11}$ Lediglich für die "alchemistischen“ Laboratorien vor 1700 liegen ausführlichere Studien von Pamela Smith (1994: 228-246, 2000, 2006), Rudolf Werner Soukup und Helmut Mayer (1997, Soukup 2007) sowie Jost Weyer (1992) vor, in denen sowohl die naturforschenden als auch die handwerklichen Dimensionen von Laboratorien thematisiert sind. ${ }^{12}$ Eine systematische vergleichende Analyse frühneuzeitlicher Laboratorien, die auch die Laboratorien der "Chemie“ (oder „Chymie“) des 18. Jahrhunderts einbezieht, gibt es dagegen bisher nicht. So beschreibt Smith in ihrem jüngsten Essay über frühneuzeitliche Laboratorien zwar die Verflechtung handwerklicher und „wissenschaftlicher" Ziele und Interessen, aber sie bezieht sich ausschließlich auf alchemistische Laboratorien vor 1700, die sie von den „wissenschaftlichen“ chemischen Laboratorien des 18. Jahrhunderts scharf abgrenzt. Bezogen auf das alchemistische Laboratorium von Graf Wolfgang II. von Hohenlohe stellt sie zutreffend fest:

Although Count Wolfgang called his early seventeenth-century workspace a Laboratorium, it resembled the workshops of apothecaries, metalworkers and pigment makers, among many other types of artisans, and it shared many of its components with the workplaces of metal smelters, glassmakers, and potters. 
Diese Feststellung ist eingebettet in eine exzellente Diskussion der Rolle der handwerklichen Künste für die Entstehung der empirischen Wissenschaften in der „wissenschaftlichen Revolution“, die jedoch keine genauere Untersuchung der Art und Weise einschließt, in der alchemistische Laboratorien und handwerkliche Werkstätten einander glichen. Im Anschluss daran heißt es explizit:

By the end of the seventeenth century, in contrast, the laboratory had become one of the hallmarks of the new science - the site where theories and hypotheses were purportedly tested by experiment and from which new discoveries and useful knowledge emerged. When and how the laboratory made the transition from artisanal workshop to its place as a central 'site of science' tells us much about how a new active epistemology in natural philosophy emerged in the early modern period to define a new kind of science. (Smith 2006, 292) (13 $^{13}$

Demgegenüber haben Chemiehistoriker wie Frederic L. Holmes und Ernst Homburg (Homburg 1999: 6) auf die Kontinuität der Ausstattung und Techniken alchemistischer und chemischer Laboratorien bis ans Ende des 18. Jahrhunderts aufmerksam gemacht. „Until late in the eighteenth century“, so Holmes, „no major technological changes altered the character of the chemical laboratory as a material or social setting." (Holmes 1989: 18) $)^{14}$

Im vorliegenden Aufsatz wird einem besonderen Typus gewerblicher Laboratorien des 18. Jahrhunderts nachgegangen, den Apothekerlaboratorien, sowie den Übereinstimmungen ihrer Ausstattung mit akademischen Laboratorien. Im Zentrum steht dabei das Laboratorium der Königlich Preußischen Akademie der Wissenschaften. ${ }^{15}$ Die lokalen historischen Umstände der Ausstattung dieses Laboratoriums mit Instrumenten, Gefäßen und Materialien belegen die Übereinstimmungen von akademischen Laboratorien und Apothekerlaboratorien des 18. Jahrhunderts in ungewöhnlich deutlicher Weise. Dieser Beleg hat selbstverständlich im Hinblick auf das größere Thema der „technowissenschaftlichen Laboratorien in der Frühen Neuzeit" nur fragmentarischen Charakter und kann einen umfassenderen systematischen Vergleich der vorwiegend mit handwerklicher Produktion befassten Laboratorien und der akademischen Laboratorien des 18. Jahrhunderts nicht ersetzen. Zudem liegt der Schwerpunkt des vorliegenden Aufsatzes ausschließlich auf der materiellen Kultur der Laboratorien. Auf Experimente und deren unterschiedliche "wissenschaftliche“ oder technologische Ziele sowie andere soziale, epistemische und ontologische Aspekte frühneuzeitlicher Laboratoriumspraktiken bin ich andernorts ausführlicher eingegangen (Klein 2005, 2007b, in Vorbereitung, Klein/Lefèvre 2007).

\section{Architektur und Ausstattung von Apothekerlaboratorien und akademischen Laboratorien}

\section{Apothekerlaboratorien}

Die pharmazeutischen Laboratorien, die in einem Keller oder einem anderen brandsicheren Raum innerhalb einer Apotheke oder auch auf dem Hof 


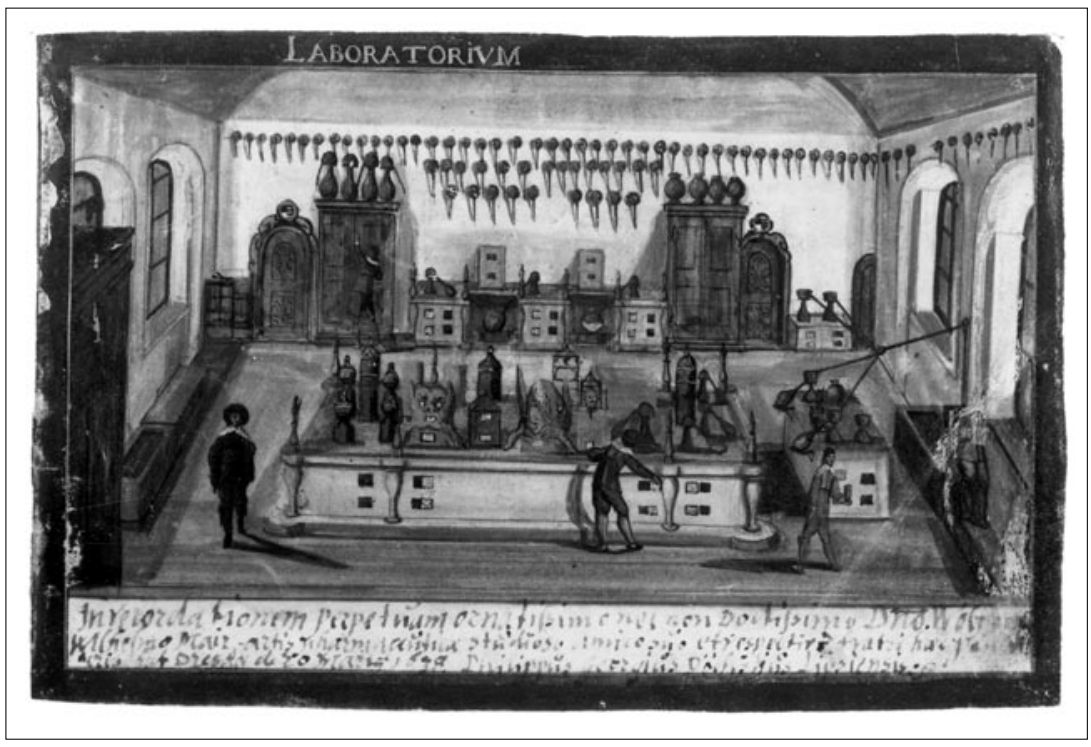

eines Apothekengebäudes eingerichtet wurden, waren zweifellos die am weitesten verbreiteten Laboratorien des 18. Jahrhunderts. In den meisten deutschen Ländern war im 18. Jahrhundert gesetzlich vorgeschrieben, dass chemische Medikamente ausschließlich von Apothekern hergestellt werden durften, und in Preußen legten Medizinalgesetze seit dem späten 17. Jahrhundert fest, dass dies in "Laboratorien“ zu geschehen habe. ${ }^{16}$ Insbesondere war es den Apothekern gesetzlich untersagt, chemische Medikamente von Materialhändlern („Materialisten“), „Laboranten“ oder anderen Produzenten und Händlern zu kaufen. Bei den „chemischen Medikamenten“ handelte es sich in der Mehrzahl nicht um chemische Syntheseprodukte im heutigen Sinn, sondern um Stoffe, die mittels chemischer Methoden wie Extraktion, Decoctionen (Auskochungen), Destillation, Sublimation, Kalzination, Auflösung und Präzipitation (Niederschlagen eines Stoffs aus seiner Lösung) aus pflanzlichen, tierischen oder mineralischen Materialien gewonnen wurden. Die Pharmakopöen des 18. Jahrhunderts listeten Hunderte dieser „chemischen Medikamente" auf, darunter zum Beispiel Oleum Camphorae, Aqua Melissae, Acetum distillatum, Spiritus Tartari, Spiritus Nitri, Crocus Martis, Flores Antimonii, Lac Sulphuris, Mercurius praecipitatus albus, Mercurius dulcis, Oleum Arsenici, Vitriolum Martis, Saccharum Saturni, diverse Elixira composita oder Laudanum opiatum. ${ }^{17}$ Destillationen und Auskochungen waren zwar schon vor dem 16. Jahrhundert im Apothekergewerbe eingeführt worden, aber erst im Kontext der paracelsischen iatrochemischen Bewegung des 16. Jahrhunderts kam es zu einer großen Verbreitung dieser Techniken. Eine stärkere Ausdifferenzierung des Angebots chemischer Medikamente setzte erst im 17. Jahrhundert ein, und in diesem Zusammenhang wurden
Abb. 1: "Laboratorium" des 17. Jahrhunderts (aus dem Stammbuchblatt von Philipp Georg Dietrich aus Leipzig für den Pharmaziestudenten Wolfram Wilhelm Mair, Dresden 1638 Mrz 20, Germanisches Nationalmuseum Nürnberg, Sign HB 13053a. Ich danke Christoph Meinel für die freundliche Weitergabe dieser Abbildung). 
Abb. 2: Chemisch-pharmazeutische Instrumente, 17.-19. Jahrhundert (mit freundlicher Genehmigung des Deutschen Apotheken-Museums Heidelberg).

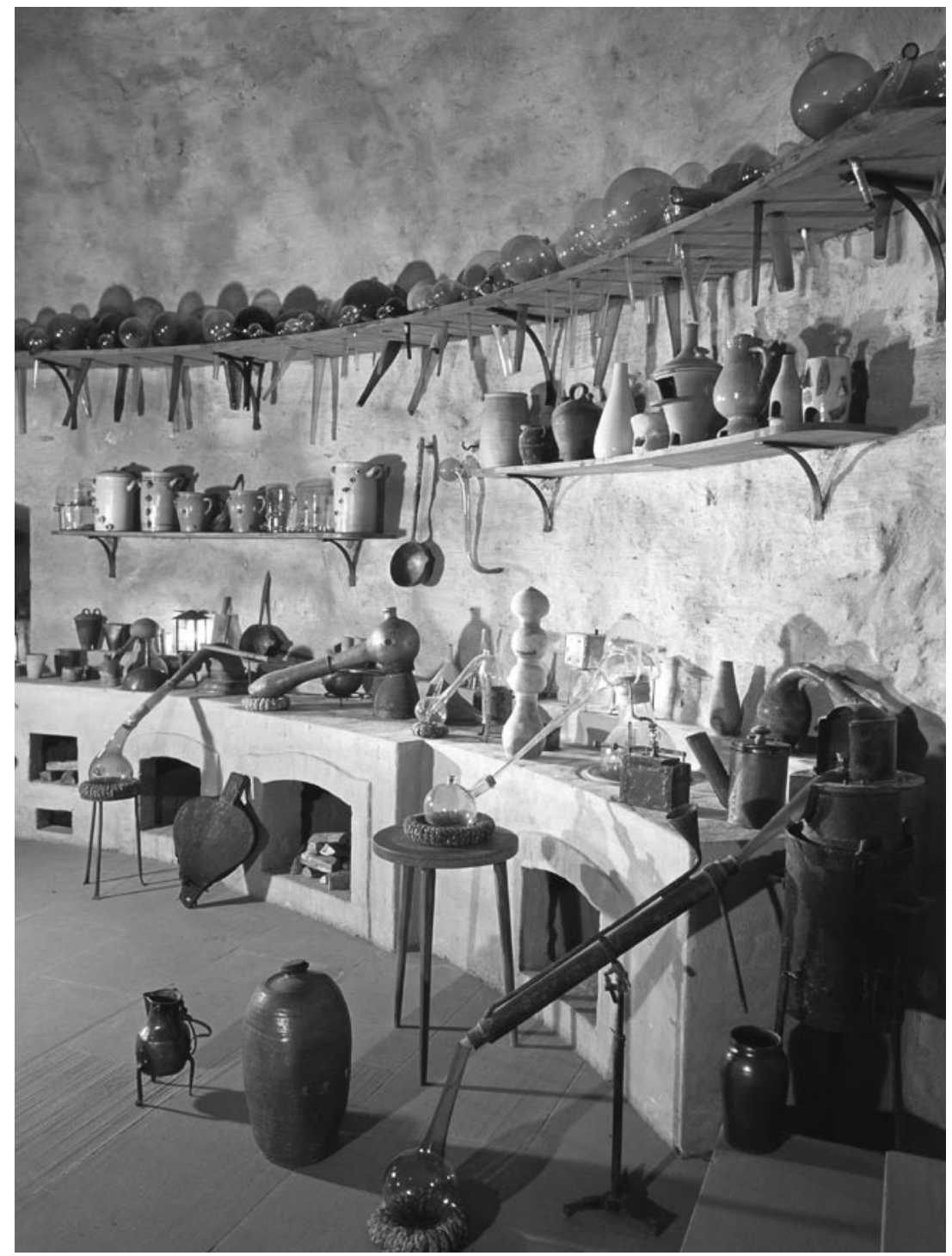

dann in größerem Umfang spezielle Räume für ihre Produktion errichtet, die Laboratorien (siehe Abb. 1). Im 18. Jahrhundert ist der Terminus „Laboratorium“ im Kontext des Apothekergewerbes allgegenwärtig und kommt in Pharmakopöen, Arzneibüchern und Beschreibungen von Apotheken sowie in Briefen, Autobiographien und wissenschaftlichen Veröffentlichungen von Apothekern immer wieder vor. Pharmazeutische Laboratorien waren selbstverständlich in erster Linie Produktionsstätten für chemische Medikamente, die in der Apotheke verkauft wurden. Im 17. und 18. Jahrhundert waren sie 


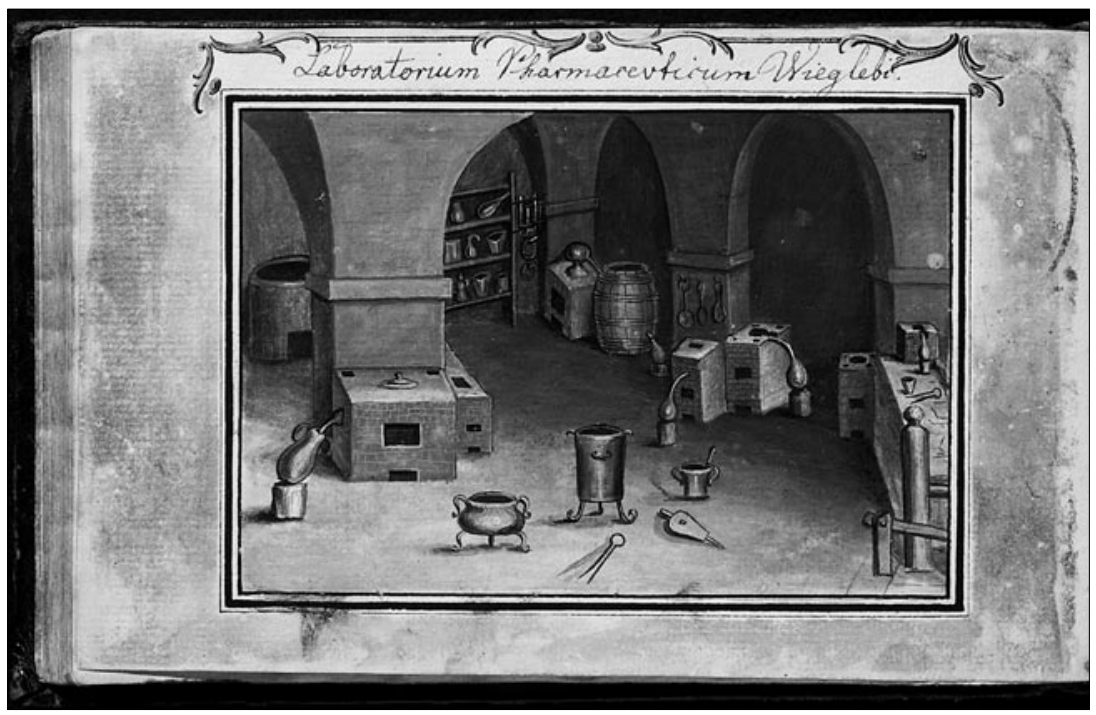

aber auch Orte der Naturbeobachtung und Forschung und zuweilen auch der Lehre. Letzteres war bei der Berliner Hofapotheke der Fall, in deren Laboratorien der praktische Chemieunterricht des Collegium medico-chirurgicum stattfand, und bei den chemisch-pharmazeutischen Schulen, die gegen Ende des 18. Jahrhunderts in Deutschland errichtet wurden. ${ }^{18}$

Ein Vergleich zwischen Apothekerlaboratorien und akademisch-chemischen Laboratorien des 18. Jahrhunderts lässt sich anhand verschiedener Quellen vornehmen. Schriftliche Quellen sind nur eine Möglichkeit, und in dieser Hinsicht ist es aufschlussreich, dass zum Beispiel die Krünitzsche Oeconomische Encyclopädie chemische und pharmazeutische Laboratorien in einem Atemzug und ohne jede weitere Differenzierung anführt. Da heute nur wenige Apothekerlaboratorien aus der Frühen Neuzeit erhalten sind, geben Apothekenmuseen am sinnfälligsten Auskunft über die pharmazeutischen Laborgefäße und -instrumente dieser Zeit. Das „Deutsche Apotheken-Museum" in Heidelberg beherbergt eine große Sammlung, in der verschiedene Destillationsapparaturen, Aludeln für die Sublimation, tragbare Öfen und andere chemisch-pharmazeutische Instrumente und Gefäße zu besichtigen sind (siehe Abb. 2). Zu den heute noch architektonisch erhaltenen Apothekerlaboratorien in Deutschland gehört das im frühen 17. Jahrhundert errichtete Laboratorium der Adler- und Ratsapotheke in Quedlinburg, das noch heute als Laboratorium benutzt wird. Aus Zeichnungen von Apothekerlaboratorien, wie der Berliner Hofapotheke oder des Laboratoriums von Johann Christian Wiegleb (1732-1800, siehe Abb. 3), die ich andernorts ausführlicher diskutiert habe, sind ebenfalls wertvolle Informationen zu entnehmen. ${ }^{19}$ Genau die gleichen Instrumente wie die in der Heidelberger Sammlung ge-
Abb. 3: Das ApothekerLaboratorium von Johann Christian Wiegleb aus dem 18. Jahrhundert (Stammbuch des Apothekergesellen J. F. Pfister, mit freundlicher Genehmigung des Deutschen Apotheken-Museums Heidelberg). 
Abb. 4: Laboratorium der Universität Altdorf (aus Puschner um 1720 mit freundlicher Genehmigung der Niedersächsischen Universitätsbibliothek Göttingen).

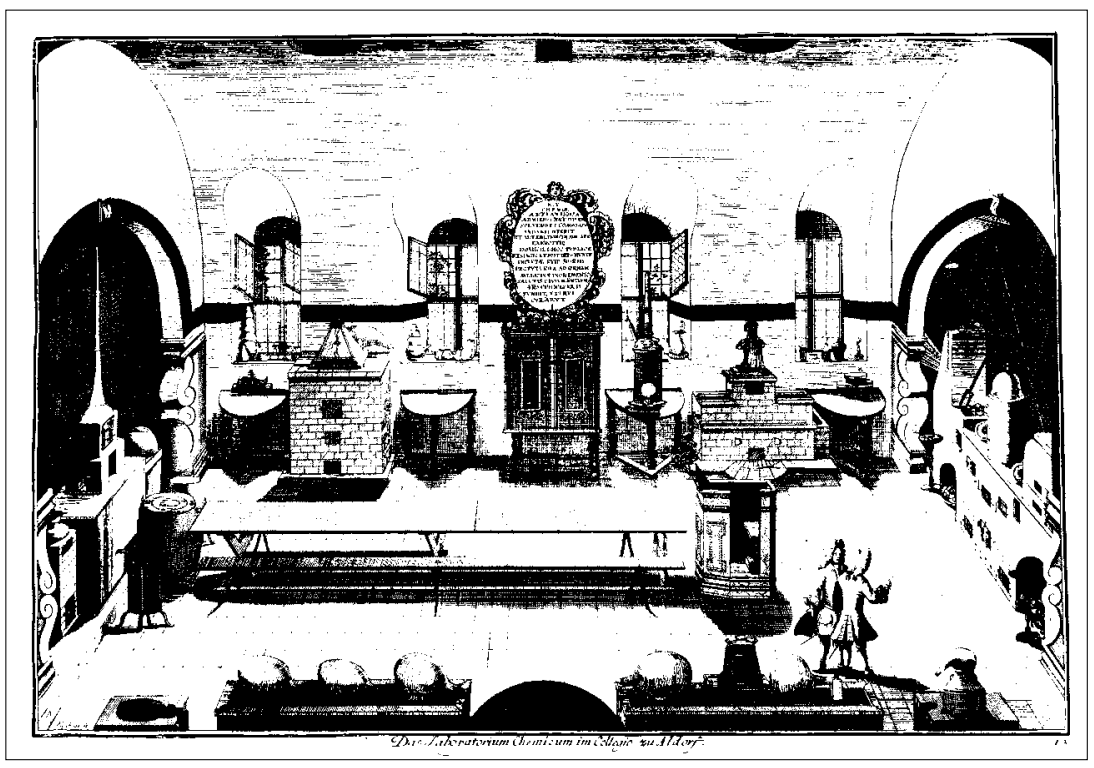

zeigten wurden auch in den akademischen Laboratorien der Zeit verwendet (siehe Abb. 4, 5). Vergleiche mit den zeitgenössischen (allerdings spärlichen) Zeichnungen von akademisch-chemischen Laboratorien zeigen ebenfalls große Übereinstimmungen beider Laboratoriumsarten. Auch Autobiographien von Apothekern sowie Veröffentlichungen im Kontext der Debatte über die Reform der Apothekerlehre im 18. Jahrhundert enthalten interessante Details über Apothekerlaboratorien (Klein 2007b). Schließlich sind hier auch die Apothekerbücher zu nennen, aus denen ich an dieser Stelle ein Beispiel anführen möchte.

Das Lehrbuch der Apothekerkunst (1778) von Karl Gottfried Hagen (1749-1829), das zu den im 18. Jahrhundert am häufigsten gelesenen deutschen Apothekerbüchern gehört, enthält eine ausführliche Beschreibung von Apothekerlaboratorien und der in ihnen verwendeten Gefäße und Instrumente. Diese Beschreibung fasse ich im Folgenden zusammen und vergleiche sie dann mit einer zeitgenössischen Beschreibung eines akademischen Laboratoriums. „Eine der vornehmsten Anstalten“, so beginnt Hagen seine Ausführungen, „ist ein Laboratorium, welches ein zu den pharmazeutischen Arbeiten besonders eingerichtetes Zimmer ist, dessen keine Apotheke leicht entbehren kann." (Hagen 1786: 23) Es folgen Anmerkungen zur Architektur des Laboratoriums, nämlich dass es "ganz gewölbt und also feuerfest" sein muss und dass es einen „wohlziehenden Schornstein“ haben muss, „damit der beschwerliche Rauch des Feuers und die schädlichen Dämpfe leicht weggeschafft werden“ (ebd.). Hagen hebt weiterhin hervor, dass für das Laboratorium ein trockener Raum ausgewählt werden muss und dass fließendes 
Wasser in der Nähe sein soll. Ferner verlangt er, dass „es mit den nöthigen Oefen, Gefässen und Instrumenten versehen sey“. Die Öfen, so fügt er hinzu, „bauet man gemeiniglich rund um an den vier Wänden des Laboratoriums, und $\mathrm{zu}$ den Gefässen werden hin und wieder Mauerspinden angebracht" (ebd.). Dann geht er ausführlich auf die verschiedenen Typen von fest eingebauten und beweglichen Öfen ein, die Blickfang eines jeden Laboratoriums im 18. Jahrhundert waren.

Dem folgen Beschreibungen der wichtigsten Gefäße wie Retorten, Destillationskolben, Scheidekolben, Rezipienten und Vorstöße für die Destillation, Alembiks (Helme für Destillationsgefäße) und Schmelztiegel. Außer Destillationen, Auskochungen und anderen chemischen Operationen in Auflösungen wurden im Apothekerlaboratorium auch die in der Metallurgie und beim metallurgischen Probieren vorherrschenden trocken-chemischen Operationen durchgeführt. Die Schmelztiegel, erklärt Hagen, braucht man „vornämlich zum Schmelzen, Kalziniren, Verpuffen und Verglasen der Metalle, Salze und anderer Substanzen" (ebd.: 34). Er empfiehlt insbesondere die in Hessen aus reinem Sand und Ton gefertigten Tiegel, doch verweist er darauf, dass man die Schmelztiegel auch „bey Waldenburg in Sachsen und bey Skele, Burgen und in anderen Gegenden von Magdeburg" kaufen kann (ebd.). Auf die übrigen Gefäße, „worinnen sowohl die Arzneien aufbehalten als auch bereitet werden“, geht Hagen nicht im Detail ein. Er setzt wohl voraus, dass seine Leser wussten, dass er hier Filter, Schalen, Gläser, Phiolen und Flaschen im Sinn hatte, die es in jedem Apothekerlaboratorium zu Dutzenden und Hunderten gab und die in Glashütten oder bei Händlern gekauft werden konnten. Ein wichtiges Anliegen Hagens war jedoch das Material, aus dem diese Gefäße gefertigt waren:

Sie müssen von einer solchen Materie verfertigt seyn, die von der darin enthaltenen Arzney keineswegs angegriffen oder aufgelöset und hiedurch verändert wird. Aus dieser Ursache sind die Gefässe von Silber, Porzellan, Glas und Steinzeug am vorzüglichsten, weil dieselben nicht leicht angegriffen werden, und wenn es auch geschehen sollte, der Arzney keine schädlichen Kräfte mittheilen können. (Ebd.: 35)

Hagen spricht hiermit ein Problem an, das zahlreiche Apotheker beschäftigte: die Verfälschung (oder Kontaminierung) von Medikamenten mit metallischen und anderen Giftstoffen. Ein Problem, das sie nicht zuletzt auch veranlasste, ihre chemischen Stoffkenntnisse zu vertiefen (Klein, in Vorbereitung).

\section{Akademische Laboratorien}

Die ausführlichste verbale Beschreibung eines akademischen (chemischen) Laboratoriums im 18. Jahrhundert stammt von dem französischen Chemiker Pierre Joseph Macquer (1718-1784), der in seinem berühmten chemischen Wörterbuch eine Art Anleitung für die Einrichtung eines solchen Laboratoriums gibt. ${ }^{20}$ Dass es sich bei seiner Beschreibung nicht um lokale Besonderheiten französischer Laboratorien handelt geht unter anderem dar- 
aus hervor, dass Macquers Ausführungen Eingang in Krünitz' Oeconomische Encyclopädie fanden, so dass ich im Folgenden gelegentlich auch auf dieses Werk zu sprechen komme. In der Einleitung seines Wörterbuchartikels betont Macquer zunächst die unbedingte Notwendigkeit eines Laboratoriums in der Chemie. ${ }^{21}$ Die darauf folgende Klarstellung, er befasse sich nur mit dem Laboratorium für gelehrte Chemiker („chymiste physicien“) setzt die weitgehende Übereinstimmung handwerklicher und akademischer Laboratorien voraus, um dann die feineren Unterschiede zu betonen. Dabei geht es insbesondere um Unterschiede in der Größenordung zwischen chemischen Experimenten und der handwerklichen Produktion sowie um die stärkere Spezialisierung einiger Typen handwerklicher Laboratorien im Vergleich zu der umfassenderen Ausrüstung, die für die „Ausübung dieser Wissenschaft“ erforderlich war. In den Worten Macquers:

Ich habe in diesem Artikel nicht die Absicht, von den zu Operationen im Großen bestimmten Laboratorien, auch nicht von denen, welche nur für einen besonderen Zweig der Chymie, z. B. für die Probirkunst, für die Schmelzgläserbereitung u. s. w. angelegt worden sind, sondern bloß von derjenigen Art von einem Laboratorium zu reden, welches ein physischer Chymist ["Chymiste Physicien“] haben muß, um, so wie es die Gelegenheit mit sich bringt, jede chymische Arbeit im Kleinen zu machen. (Macquer 1789, 4: 2) ${ }^{22}$

Was die Architektur eines akademischen Laboratoriums anbetrifft, so empfiehlt Macquer, dieses im Erdgeschoss oder ersten Stockwerk einzurichten sowie in Räumen, die ein Steingewölbe besitzen. Beides war, wie bereits aus dem weiter oben diskutierten Apothekerbuch Hagens hervorging, auch für Apothekerlaboratorien üblich. Steindecken, die aus statischen Gründen stets gewölbt waren, dienten dem Feuerschutz, denn angesichts der zahlreichen Öfen war die Brandgefahr hoch. Das Erdgeschoss war vor allem deshalb ein geeigneter Ort, weil große Mengen Holz oder Kohle für die Öfen benötigt wurden; zusätzlich war das Wasser für das Reinigen der Gefäße am bequemsten im Erdgeschoss zu beschaffen. Aus beiden Gründen wurden auch Kellergewölbe als Laboratorien ausgestattet, aber hier bereitete die Raumfeuchtigkeit Probleme:

Viele Leute stehen in der Meynung, dass ein Laboratorium unten auf der Erde, vornehmlich wegen des Wassers, des Waschens und Spülen u.s.w. bequember sey; und es hat dieser Betrachtung seine Vortheile; auf der anderen Seite aber hat es auch, vornehmlich der Feuchtigkeit wegen, sehr große Unbequemlichkeiten. Die immerwährende Feuchtigkeit wird, ohnerachtet sie sogar für viele Gegenstände unmerklich und sehr beträchtlich ist, bey einem chymischen Laboratorium eine sehr große Beschwerlichkeit. An einem solchen Orte werden die meisten salzartigen Materien mit der Zeit feucht, die Aufschriften lösen sich ab, beschlagen oder verlöschen, die Blasebälge gehen zu Grunde, die Metalle rosten, die Oefen werden unbrauchbar, kurz fast alles verdirbt. (Ebd.: 2 f.)

Mindestens ebenso wichtig wie ein trockener Raum war eine gute Belüftung, was meist durch einander gegenüber liegende Fenster erreicht wurde. Dies war wegen der „Fortschaffung der gefährlichen Dämpfe oder des gefährlichen Staubs mancher Substanzen (,drogues') sehr nöthig“ (ebd.: 3). Mac- 
quers Beschreibung der Architektur eines akademischen Laboratoriums vermittelt bereits einen guten Eindruck von einem akademischen Ort, an dem Materialität sehr handgreiflich zu erfahren war und der einer handwerklichen Werkstätte nicht unähnlich war. Dies wird noch deutlicher in seinen Anweisungen für den Bau des Rauchfangs und des Schornsteins mit Feuermauer sowie der detaillierten Beschreibung der zahlreichen Öfen, von denen die größeren fest eingemauert wurden. In der Krünitz‘schen Oeconomischen Encyclopädie füllen diese Anweisungen mehrere Seiten und werden ergänzt durch ausführliche Belehrungen über die Feuergefahren im Laboratorium (Krünitz 1773-1858, 242: 50-54). Die Destillieröfen, Schmelzöfen, Lampenöfen, Reverberieröfen und Probieröfen sowie die Blasebläge, Schürhaken, Gabeln und Zangen, die man für ihre Befeuerung brauchte, dominierten alle Unterarten von Laboratorien im 18. Jahrhundert und in den Jahrhunderten davor. Das Holz und die Kohlen, von denen man stets „einen Vorrath bey der Hand haben" musste, wurden in einer Ecke des Laboratoriums gelagert oder besser noch in einem angrenzenden Vorratsraum, da der schwarze Kohlenstaub „eine beständige Ursache von Unreinlichkeit“ war (Macquer 1789, 4: 6). In einem zusätzlichen Vorratsraum wurden noch andere Materialien aufbewahrt, die man in größeren Mengen benötigte, wie Ton, Kalk, Sand, Backsteine und Ziegel. Wasser wurde in einem Fass oder Trog in der Ecke des Laboratoriums bereitgestellt.

Die verschiedenen kleineren Glasgefäße, die zum Beispiel für Auflösungen, Präzipitationen und Filtrationen sowie für das Aufbewahren von Reagenzien und chemischen Produkten verwendet wurden, befanden sich in Regalen oder Simsen in der Wand und zuweilen auch in Schränken. „Diese Gesimse müssen so vielfach als möglich seyn“, bemerkt Macquer, „indem man in einem Laboratorium, worinnen man fleissig arbeitet, von denselben, so zu reden, niemals genug haben kann." (Ebd.: 5). Die Arbeiten, von denen hier die Rede ist, wurden auf einem großen Tisch in der Mitte des Laboratoriums durchgeführt. Auf diesem Tisch wurden „die Vermischungen, die Vorbereitungen zu den Arbeiten, die Auflösungen, Niederschlagungen, Durchseihungen im Kleinen, kurz alles das verrichtet, was keine Beyhülfe des Feuers erfordert, außer etwa blos das Lampenfeuer" (ebd.: 5f.). Unter den zahlreichen anderen Gefäßen und Instrumenten eines chemischen Laboratoriums - in der deutschen Übersetzung ist hier interessanterweise von „kleinem Hausrath“ und anderen kleinen Gerätschaften die Rede - nennt Macquer unter anderem Mörser, Hammer, Scheren und Reiben, Kapellen (flache, aus Knochenasche bestehende Schalen) und Muffeln (Abdeckglocken) für Schmelzarbeiten und das Probieren von Metallen, Filter und Filterpapier, Strohhalme und gläserne Röhren zum Umrühren, Korkstöpsel, Blasen und Leinenstreifen zum Verschließen von Geräten sowie verschiedene Waagen und Gewichte bis hin zu Schreibpapier (ebd.: 7). Ein weiterer Blickfang eines jeden chemischen Laboratoriums im 18. Jahrhundert wa- 


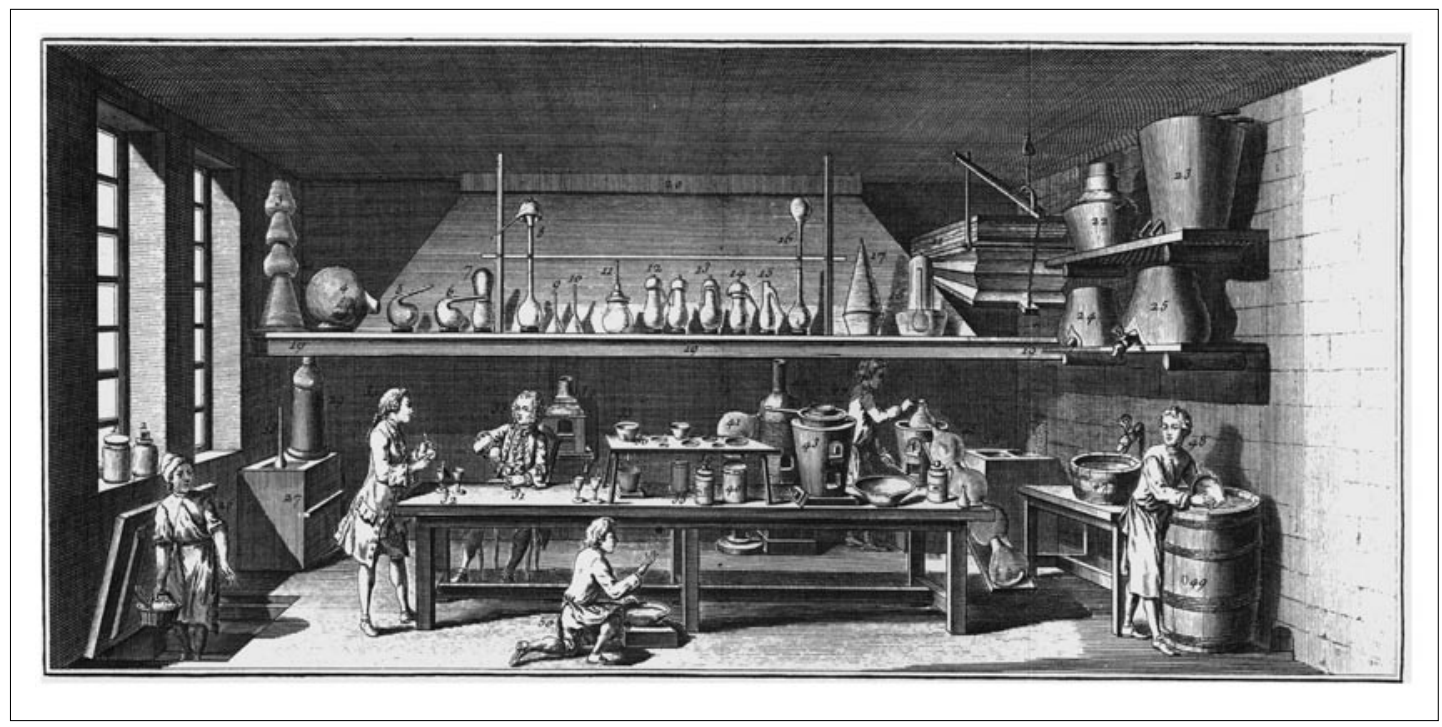

Abb. 5: Chemisches Laboratorium des 18 Jahrhunderts (aus Diderot/ d'Alembert 1966, XXIV Chemie Planche 1). ren die Destilliergefäße wie Retorten, Alembiks und Kolben unterschiedlicher Größe und Materialien. Die birnenförmigen, tönernen Aludeln zum Sublimieren, die Phiolen und Glasflaschen mit Glasstöpseln, Arzneigläser, Schachteln und eine Reihe anderer Gefäßarten, für deren detaillierte Beschreibung Macquer einen eigenen Artikel "Gefäße“ in sein Wörterbuch aufnahm, gehörten ebenfalls zur normalen Ausstattung eines akademischen Laboratoriums (siehe Abb. 4, 5).

Alle diese Gefäße waren im Handel relativ kostengünstig erhältlich - ganz im Gegensatz zu vielen „philosophischen Instrumenten“ der Zeit - und alle diese Gefäße wurden auch in Apothekerlaboratorien verwendet. Krünitz fügt noch die interessante Bemerkung hinzu, die Gefäße, die man in einer „chemischen Werkstatt" brauche, seien „einfach“ - er erwähnt unter anderem auch Flaschen, Zuckergläser und Arzneigläser - und man verwende „weniger gekünstelte Gefäße“ als die Alchemisten in den Jahrhunderten zuvor (Krüntiz 1773-1858, 242: 58). Zwischen den alchemistischen Laboratorien des 16. und 17. Jahrhunderts und den chemischen Laboratorien des 18. Jahrhunderts bestand zwar eine große Kontinuität (Holmes 1989, Homburg 1999), jedoch wurden mehrere Geräte aus der alchemistischen Periode im 18. Jahrhundert nur noch selten verwendet. Das betrifft beispielsweise die hermetisch abgeschlossenen „philosophischen Eier“ (für die Herstellung des Steins der Weisen), Pelikane (geschlossene Gefäße zum wochenlangen Zirkulieren der Stoffe) und den speziellen Glasapparat für die Herstellung des Paracelsischen Clissus, der noch auf der berühmten Abbildung eines chemischen Laboratoriums in Diderots Encyclopédie zu sehen ist (siehe 
Abb. 5, rechte hintere Ecke). Stattdessen kamen von Mitte des 18. Jahrhunderts an allmählich neue „philosophische Instrumente“ hinzu. ${ }^{23}$ Diese allmähliche Veränderung in der materiellen Kultur des akademischen Laboratoriums kommt in der zweiten Ausgabe von Macquers Wörterbuch von 1778 zum Ausdruck, die gegenüber der ersten Ausgabe von 1766 eine Reihe neuer Instrumente anführt, darunter Gefäße zum Auffangen von Gasen sowie "Maschinen und Instrumente, die man zuvor nur in physikalischen Kabinetten (cabinets de physique) beobachten konnte" (Macquer 1778, 2: 6). ${ }^{24} \mathrm{Zu}$ letzteren gehörten Thermometer, Barometer, Brenngläser, Lupen und Mikroskope, Luftpumpen und Elektrisiermaschinen. Alle diese neuen Instrumente, so Macquer, mussten in einem speziellen Raum in der Nähe des chemischen Laboratoriums aufbewahrt werden, „weil sie sonst in kurzer Zeit durch die Dämpfe, die in den meisten Operationen aufsteigen, verderbt werden würden" (ebd.). Die Integration philosophischer Instrumente in chemische Laboratorien war ein langsamer Prozess, der unter anderem auch von den vorhandenen finanziellen Mitteln abhing. Er beinhaltete eine Ausdifferenzierung von akademischen und handwerklichen Laboratorien im Verlauf des 18. Jahrhunderts, die jedoch vor dem 19. Jahrhundert noch nicht zu einer Separierung führte. Dies war unter anderem deshalb nicht der Fall, weil auch in Apothekerlaboratorien, Laboratorien von Probierern und anderen handwerklich-gewerblichen Laboratorien derartige Instrumente übernommen wurden.

In seiner Beschreibung der Ausstattung von Laboratorien geht Macquer auch auf die "Materialien“ ein, die für chemische Operationen gebraucht wurden. Die französische Originalausgabe benutzt hier das Wort „drogues“, das ebenso wie „Materialien“ auf die gewerbliche Verwendung dieser Stoffe verweist. Die übergroße Mehrzahl der Stoffe, die in den akademischen Laboratorien des 18. Jahrhunderts als Reagenzien sowie als Untersuchungsobjekte verwendet wurden, stammte aus der damaligen Warenwelt (Klein/Lefèvre 2007). Insbesondere bei den Stoffen pflanzlicher und tierischer Herkunft handelte es sich in der Regel um Waren, die aus Übersee importiert oder in einheimischen Apotheken gewonnen wurden. Macquers Beschreibung der einzelnen Stoffe lässt keinen Zweifel an ihrer gewerblichen Herkunft. So nennt er zuerst "Gemeine Vitriolsäure, wie man sie bey Materialhändlern findet", es folgt "gemeines und wohlfeiles Scheidewasser, wie man es bey Scheidewasserbrennern findet", und dann "Salzgeist, wie ihn die Scheidewasserbrenner liefern" (Macquer 1766, 2: 8, 1789, 4: 13). Auch im Laboratorium der Königlich Preußischen Akademie der Wissenschaften wurde mit zahlreichen aus dem Handwerk bekannten Stoffen experimentiert, die von Apotheken geliefert wurden. Aber auch hinsichtlich der Ausrüstung des Berliner Akademielaboratoriums mit Instrumenten und Gefäßen zeigt sich eine große Übereinstimmung mit Macquers und Krünitz' Beschreibungen. 


\section{Das Laboratorium der Königlich Preußischen Akademie der Wissenschaften zu Berlin}

Pläne für den Bau eines Laboratoriums für die Königlich Preußische Akademie der Wissenschaften zu Berlin (beziehungsweise die Societät der Wissenschaften, ihrem Vorläufer von 1700-1744) lassen sich bis in die 1720er Jahre zurück verfolgen. Erst 1753 jedoch, unter Friedrich dem Großen, nahmen diese Pläne konkrete Gestalt an. Im Juni 1753 fasste die Akademie den Beschluss, ein Laboratoriumshaus zu errichten, in dessen oberem Stockwerk auch eine Wohnung für den Direktor des Laboratoriums untergebracht werden sollte. Der Baubeginn war im Sommer 1753 auf einem der Akademie gehörenden Gartengelände in der Dorotheenstraße 10, das direkt gegenüber dem Observatorium und dem Hauptgebäudekomplex der Akademie gelegen war (hinter der heutigen Humboldt-Universität). ${ }^{25}$ Im Frühjahr 1754 war das Gebäude einzugsbereit für den ersten Direktor des Laboratoriums, den Apotheker und Chemiker Andreas Sigismund Marggraf (1709-1782), den die Akademie ein Jahr zuvor mit königlicher Billigung zum Direktor ernannt hatte. Als Laboratoriumsdirektor war Marggraf auch verantwortlich für die Ausstattung des Laboratoriums, von den fest installierten Öfen, die im Zuge des Hausbaus errichtet wurden, abgesehen.

Die besonderen Umstände der Ausstattung dieses Laboratoriums sind ein historischer Glücksfall, weil sie ungewöhnliche Aufschlüsse über die Beziehung zwischen akademischen und pharmazeutischen Laboratorien im 18. Jahrhundert geben. Aus den Dokumenten der Preußischen Akademie der Wissenschaften geht nämlich eindeutig hervor, dass Marggraf das Akademielaboratorium nicht durch Neuankauf oder Spezialanfertigung von Geräten und Instrumenten quasi vom Nullpunkt an ausstattete, sondern die Geräte und Instrumente aus dem eigenen Apothekerlaboratorium verwendete. ${ }^{26}$ Es gab in diesem Fall also einen direkten Transfer chemischer Geräte und Instrumente vom Apothekerlaboratorium ins akademische Laboratorium. Aus dem Inventarium dieser Instrumente, das weiter unten diskutiert wird, ist weiterhin zu ersehen, dass Marggrafs Apothekerlaboratorium nicht etwa anders oder besser ausgestattet war als die üblichen Apothekerlaboratorien der Zeit. Es ist also keineswegs so, dass der Apotheker-Chemiker Marggraf, der in seinem Apothekerlaboratorium auch chemische Experimente durchführte, über die er in den Miscellanea Berolinensia und den Memoiren der Akademie berichtete, eine bessere, für damalige Apotheken untypische Laborausrüstung besessen hätte.

Zunächst aber einige Worte über Marggraf selbst, mit dessen Karriere und Charakterisierung als Apotheker-Chemiker ich mich andernorts schon ausführlicher beschäftigt habe. ${ }^{27}$ Marggraf begann im Alter von etwa 16 Jahren in der Berliner Apotheke seines Vaters eine Apothekerlehre, die er 1726 an der Königlichen Hofapotheke in Berlin fortsetzte. Die Königliche Hofapo- 
theke wurde zu dieser Zeit von dem Apotheker-Chemiker Caspar Neumann (1683-1737) geleitet, der gleichzeitig Professor für Chemie an dem kurz zuvor für die höhere Ausbildung von Chirurgen gegründeten Collegium medico-chirurgicum war. Neumanns experimentelle Instruktionen im Rahmen des Chemieunterrichts an der neuen Chirurgenschule fanden im Laboratorium der Königlichen Hofapotheke statt, die in einem Seitenflügel des Berliner Schlosses gelegen war. Der Ort von Marggrafs Lehre, und vermutlich auch die Lehre selber, war somit nicht ganz typisch für eine Apothekerlehre. Nachdem Marggraf 1731 die Lehre abgeschlossen hatte, begannen die üblichen Wanderjahre der Gesellenzeit. Marggraf verbrachte zunächst ein Jahr in einer Apotheke in Frankfurt am Main und ein weiteres Jahr in einer Straßburger Apotheke. Dann ging es weiter in die Universitätsstadt Halle, wo er Vorlesungen in Medizin und Chemie hörte. Dieses Intermezzo an einer Universität dauerte jedoch nur ein knappes Jahr. Die nächste Station war die Bergbaustadt Freiberg, in der der berühmte Bergrat Johann Friedrich Henckel (1678-1744) Mineralogie, Metallurgie und metallurgische Probierkunst unterrichtete. 1735 kehrte Marggraf dann auf einem Umweg durch die Bergbauregionen des Harzes nach Berlin zurück und wurde dort Provisor der Apotheke seines Vaters, eine Tätigkeit, die er fast zwanzig Jahre ausübte.

Bereits 1738, nur drei Jahre nach seiner Rückkehr nach Berlin, erhielt Marggraf mit seiner Wahl zum ordentlichen Mitglied der Berliner Societät der Wissenschaften (ab 1744 Akademie der Wissenschaften) seine erste Anerkennung als Chemiker. Dies ist nicht zuletzt auch deshalb erstaunlich, weil Marggraf bis zu diesem Zeitpunkt noch keine Veröffentlichungen vorzuweisen hatte. Marggrafs erster Experimentalbericht erschien erst zwei Jahre später, und danach setzte eine rege Publikationstätigkeit ein, fast ausschließlich in den Miscellanea Berolinensia der Societät der Wissenschaften. Daneben hielt Marggraf auch regelmäßig öffentliche Vorträge über Chemie. Marggrafs Veröffentlichungen und Vorträge in der Zeit von 1735 bis 1754 basierten allesamt auf Experimenten, die er in seinem Apothekerlaboratorium durchgeführt hatte, darunter auch sein berühmtestes Experiment zur Extraktion von Zucker aus Zuckerrüben. Nach jahrelanger praktischer Tätigkeit als Apotheker erhielt Marggraf 1753 das Angebot, Direktor des neu zu errichtenden chemischen Laboratoriums der Preußischen Akademie der Wissenschaften zu werden. Dieses Angebot kam just zu dem Zeitpunkt, als die Apotheke des Vaters aus familiären Gründen verkauft werden musste. Dem folgte 1760 die Ernennung zum Direktor der Physikalischen Klasse der Akademie, eine Position, die Marggraf bis zu seinem Tod innehatte. Dabei sollte nochmals betont werden, dass dieser Direktor des Laboratoriums und der Physikalischen Klasse der Preußischen Akademie der Wissenschaften nur eine Apothekerlehre abgeschlossen hatte. Aber er war in dieser Hinsicht keine Ausnahme, denn seine beiden Nachfolger, Franz Carl Achard (17531821) und Martin Heinrich Klaproth (1743-1817), hatten ebenfalls kein Uni- 
versitätsstudium absolviert. Klaproth war auch Apotheker und Chemiker, und Achard hatte sich auf individuellen Wegen und teilweise durch Selbststudium einen Namen als Chemiker und Naturkundler erworben. Die wichtigste Voraussetzung für die hybride Persona eines Apotheker-Chemikers wie Marggraf und Klaproth war die Existenz pharmazeutischer Laboratorien und die große Übereinstimmung der materiellen Kultur akademisch-chemischer und pharmazeutischer Laboratorien. Beides ermöglichte fließende Übergänge von der Herstellung chemischer Medikamente und der technologischen Untersuchung von Herstellungsprozessen zu chemischen Analysen und anderen Erkundungen der stofflichen Natur (und umgekehrt).

\section{Die Ausstattung des Akademielaboratoriums}

Etwa ein Jahr nach seinem Einzug in das akademische Laboratoriumshaus stellte Marggraf ein „Inventarium von denen Stücken so zum Laboratorium Chymicum gehören und bis Ende 1754 angeschaft worden" zusammen (ABBAW: I-XIII-20, Blätter 1-4). ${ }^{28}$ Dieses Inventarium gibt über die wenigen Instrumente Aufschluss, die Marggraf im ersten Jahr seiner Direktorenzeit neu hinzukaufen musste. Dabei handelte es sich um einen großen Blasebalg mit Gestell, eine kupferne Blase zum Destillieren mit Zubehör, eine große Retorte, die eingemauert wurde, einen „Aufsatz“ (vermutlich für die Destillation) aus Eisenblech, zwei eiserne Kapellen, zwei eiserne Platten, zwei „Spreng“-Eisen, zwei große eiserne Mörser, ein kupfernes Marienbad, ein Tisch mit elf Schubladen und sechs Schemel. Es ist ausgeschlossen, dass diese Instrumente und Materialien für die chemischen Experimente Marggrafs im ersten Jahr seiner Direktorenzeit ausreichten. Denn wie aus seinen Berichten auf den Sitzungen der Akademie am 19. Juni 1754 sowie am 25. September und am 7. November desselben Jahres hervorgeht, führte er in dieser Zeit unter anderem Versuche zur Analyse und Resynthese von Alaunerde durch, die Operationen wie Auflösungen, Präzipitationen, Evaporationen und Kristallisationen einschlossen (Köhnke 1900: 180, Winter 1957: 201-258). Für derartige chemische Operationen wurden gewöhnlich kleinere Glaskolben, Glasbecher, Schalen und Filter sowie zahlreiche Reagenzien verwendet, die im Inventarium von 1755 jedoch nicht vorkommen. Woher stammten diese Gefäße und Materialien? Ein mehr als zehn Jahre später zusammengestelltes Inventarium beantwortet diese Frage eindeutig: aus Marggrafs Apothekerlaboratorium.

Im Zuge der Reorganisation der Finanzen der Akademie nach dem Siebenjährigen Krieg genehmigte Friedrich II. die Erneuerungen von Akademiegebäuden, darunter auch eine Renovierung und Erweiterung des akademischen Laboratoriums (Harnack 1900, 1 (1): 363, 487-92). Durch diese Erweiterung standen ab 1766 außer dem eigentlichen Laboratoriumsraum vier weitere Räume für die Aufbewahrung von Instrumenten, Gefäßen und Materialien zur Verfügung. Für Marggraf bot diese Neuorganisation eine gute Gelegenheit, sich endlich für seine persönlichen Investitionen in das 
akademische Laboratorium entschädigen zu lassen. Im Oktober 1766, als die Umbauten abgeschlossen waren, wandte er sich mit einer entsprechenden Bitte an die neu gegründete ökonomische Kommission der Akademie. Seinem Brief ist eindeutig zu entnehmen, dass Marggraf das Akademielaboratorium weitgehend mit eigenen Geräten ausgestattet hatte, denn es heißt dort wörtlich: „Es ist aus dem Archiv der Academie zu ersehen, das alles was sich im Laboratorio befindet, ein weniges davon ausgenommen, von mir dahin mitgebracht worden sey." Marggrafs Wink, seine Erben könnten im Falle seines Todes Rückgabeansprüche stellen, trug wohl nicht unerheblich zu einer umgehenden Beantwortung seines Briefs bei. Die Kommission deutete die Bereitschaft zur finanziellen Entschädigung an und bat Marggraf, eine Inventarliste für die Dinge aufzustellen, „die er der Academie zum Gebrauch des Laboratorii überlassen will“ (ebd.). Etwa vier Monate später, im Februar 1767, war diese Liste fertig. ${ }^{29}$

Unter den Dingen, die aus Marggrafs Apothekerlaboratorium stammten, befanden sich acht tragbare Öfen mit Aufsätzen, mehr als 400 Retorten unterschiedlicher Größe und unterschiedlichen Materials (darunter waren 380 irdene, zwei aus Eisen bestehende und zwanzig aus weißem und dreißig aus grünem Glas), zwei große Rezipienten für Destillationen aus grünem Glas, 144 kleinere Rezipienten, Phiolen und Kolben aus weißem oder grünem Glas, „eine Menge ganz kleiner Kolben und Retorten“, eine Apparatur („Maschine") aus Glas zur Destillation an der Sonne, zahlreiche Gläser mit und ohne gläserne Stöpsel, Kapellen von unterschiedlicher Größe sowie Tiegel, Messingscherben und weiteres Zubehör für Schmelzarbeiten, Messingpfannen und eiserne Töpfe, eine eiserne Amalgamiermaschine, Mörser, Hammer und verschiedene Ambosse. Alle diese Geräte und Instrumente wurden in den Apothekerlaboratorien des 18. Jahrhunderts verwendet und sind heute in Apothekenmuseen zu besichtigen. Darüber hinaus erwähnt das Inventarium noch zwei Tonnen weißen Ton, verschiedene "praeparata " und "Materialien“ sowie die Tische und Schemel, die Marggraf 1754 gekauft hatte.

Marggraf kalkulierte für alle diese Geräte eine Summe von rund 322 Reichstalern, die ihm wenig später auch ausgehändigt wurde. Das ist ein relativ bescheidener Betrag, wenn man ihn mit den Ausgaben für das Observatorium der Akademie vergleicht, die sich beispielsweise im Jahr 1768 auf rund 1295 Reichstaler beliefen. Im Unterschied zu den „philosophischen Instrumenten" der Zeit waren die chemischen Instrumente jedoch viel kostengünstiger, und auch die laufenden jährlichen Kosten des Laboratoriums waren mit rund 200 Reichstalern 1768 relativ niedrig. Der größte Teil (etwa 40\%) dieser laufenden Ausgaben wurde für Arbeitslöhne und Materialien verwendet, wogegen die Kosten für Instrumente und Gefäße relativ gering waren. Somit ergibt sich auch aus dieser zusätzlichen Perspektive der laufenden Finanzierung, dass das akademische Laboratorium keineswegs eine exklusive akademische Einrichtung war. 
Alles in allem zeigt der Vergleich von Marggrafs Inventarium mit Macquers Beschreibung akademisch-chemischer Laboratorien, dass das Königlich Preußische Akademielaboratorium keine Ausnahme unter den akademischen Laboratorien war. Weiterhin ergibt sich aus dem Vergleich der Ausstattung des Akademielaboratoriums mit derjenigen von Apothekerlaboratorien sowie aus dem tatsächlichen Transfer von Instrumenten und Gefäßen von Marggrafs Apothekerlaboratorium ins Laboratorium der Berliner Akademie das Bild einer großen Übereinstimmung der materiellen Kultur gewerblicher Apothekerlaboratorien und akademischer Laboratorien im 18. Jahrhundert. Das trifft nicht zuletzt auch auf die Stoffe zu, mit denen Marggraf im Berliner Akademielaboratorium experimentierte. Dokumente über Rechungen, Einnahmen und Ausgaben geben auch hier eine eindeutige Auskunft. Marggraf bezog bis 1769 die Stoffe, die er für seine Experimente benötigte, von einem Apotheker namens Fabricius, ab 1769 zusätzlich von dem bekannten Berliner Apotheker Valentin Rose Sr. (1736-1771), dem Besitzer der Apotheke Zum Weissen Schwan. ${ }^{30}$ Der materielle Transfer vom Apothekerlaboratorium zum akademischen Laboratorium, der mit der Ausrüstung des Laboratoriums begann, wurde auf diese Weise fortgesetzt.

\section{Der Bau des Laboratoriumshauses nach 1766}

Weitere Belege für die Übereinstimmungen zwischen den Apothekerlaboratorien und akademischen Laboratorien des 18. Jahrhunderts ergeben sich aus der Betrachtung des Baus des akademischen Laboratoriumshauses und seiner Grundausstattung mit Öfen und Kaminen. Im April 1768 stellte die Ökonomische Kommission der Akademie ein umfassendes Inventarium „Über die in dem Königl. Preußischen Laboratorio befindliche Sachen an Instrumenten, Oefen und Praeparaten“ zusammen. Aus diesem Inventarium geht Folgendes hervor: An der Wand rechts von der Eingangstür zum Laboratorium war ein großer Kamin mitsamt einem Blasebalg eingebaut. Unter diesem Kamin standen ein großer "General-Ofen“ und zwei kleinere Probieröfen mit Muffeln für das Schmelzen und Probieren von Metallen und anderen Mineralien. An der Wand links von der Eingangstür war ein zweiter Kamin eingebaut, unter dem sich ein Digerierofen, zwei tragbare Öfen für Destillationen und Digestionen, ein Glauberscher Ofen mit Zubehör, ein kleiner und ein großer Destillierofen und ein kleiner und ein großer WindOfen sowie diverses Zubehör für diese Öfen befanden. Insgesamt war das Akademielaboratorium mit fünfzehn Öfen ausgestattet, darunter mehrere Schmelzöfen und Destillieröfen mit fest installierten Destillationsapparaturen, die mit kupfernen oder eisernen Retorten und kupfernen Kühlern ausgestattet waren. Der obligatorische große Tisch für Auflösungen und andere chemische Operationen im Kleinen stand an der Wand zwischen den Fenstern des Laboratoriums. In den Schubladen dieses Tisches wurden kleine Glasgefäße sowie verschiedene Metall-Lösungen und Säuren, die häufig als 
Reagenzien benötigt wurden, aufbewahrt. Links neben der Eingangstür befanden sich außerdem eine Wasserzisterne sowie ein großer eiserner Mörser mit Pistill, ein großer Hammer, eine Kelle und auch die Schemel, die Marggraf 1754 gekauft hatte.

Alle anderen Instrumente, Gefäße und Materialien waren in den vier Räumen untergebracht, die bei der Erweiterung des Laboratoriumshauses von 1764-66 hinzugekommen waren. Unmittelbar an den eigentlichen Laboratoriumsraum grenzte ein Raum, der mit zahlreichen Regalen, Schränken und Tischen ausgestattet war und in dem verschiedene Gefäße, Chemikalien und unfertige chemische Präparate („chymische Arbeiten, welche noch durchgesehen werden müssen“) aufbewahrt wurden. In diesem Raum ließ man auch die gereinigten Gefäße trocknen. Weiterhin befanden sich hier Hammer und Ambosse und sogar eine Schreibtafel. Ein zweiter, als „Praeparations Cammer" bezeichneter Raum befand sich ebenfalls im Erdgeschoss gleich neben der Haupteingangstür zum Gebäude. Hier wurden verschiedene Typen von Waagen und Gewichten aufbewahrt und weitere Reagenzien, Metallplatten und andere Rohmaterialien sowie kleinere Geräte wie Spatel, Mörser und Schmelztiegel. Schließlich befand sich im Erdgeschoss auch die „Kohlen Cammer“. Die große Mehrzahl der Gefäße und Instrumente wurde in einem weiteren Raum aufbewahrt, der sich im Dachgeschoss befand. Das Inventarium listet hier Hunderte von gläsernen und irdenen Gefäßen auf, die von alltäglichen Gefäßen wie „Zucker Gläsern“, „Urin Gläsern“, „Milchnäpfen“ und „Florentiner Weinflaschen“ über Phiolen, Schmelztiegel und verschieden geformte Retorten und Destillationskolben bis zu einer „Maschine“ für die Verbrennung von Phosphor und einer für Destillationen an der Sonne reichen. Die beiden zuletzt genannten Geräte waren dabei die einzigen, die für Apothekerlaboratorien eher ungewöhnlich waren.

Auch die im zweiten Inventarium von 1768 aufgelisteten Geräte und Instrumente des akademischen Laboratoriums dürften noch größtenteils aus dem Apothekenlaboratorium Marggrafs gestammt haben. Dieser direkte und ungewöhnliche Beleg für die Übereinstimmung der materiellen Kultur von Apothekenlaboratorien und akademischen Laboratorien im 18. Jahrhundert ist jedoch kein Einzelfall. Denn ungefähr vierzig Jahre später, als der Apotheker und Chemiker Martin Heinrich Klaproth Direktor des Akademielaboratoriums wurde, wiederholte sich ein solcher materieller Transfer in nahezu in allen Facetten. Nach Marggrafs Tod im Jahr 1782 wurde Franz Carl Achard berufen. Als Nachfolger Marggrafs übernahm Achard ein gut ausgestattetes Laboratorium. Das änderte sich jedoch drastisch im Jahr 1799, als Achard das Laboratorium in eine technologische Versuchsanstalt für die Extraktion von Zucker aus Zuckerrüben umbaute, was ausdrücklich vom akademischen Direktorium genehmigt war. Der selbst gewählte Name für diese Versuchsanstalt - „Zuckerfabrik“ - enthüllt worum es ging: Nicht um eine Zuckerextraktion im kleinen experimentellen Maßstab, sondern um 
die technologische Exploration der Zuckergewinnung in der Größenordnung von Zentnern (Müller 2002). Achards technologische Versuche gingen nicht spurlos am akademischen Laboratorium vorüber und auch nicht an der darüber liegenden Wohnung. Nachdem Achard 1801 Berlin verlassen hatte, um in Schlesien eine eigene Zuckerfabrik zu bauen, fand sein Nachfolger Klaproth ein Desaster vor. In einem Brief wandte er sich an das „Königliche wohllöbliche akademische Direktorium“ mit der „dringende[n] Bitte, der schleunigen Herstellung des im Argen liegenden academischen Hauses, in einen, der Ehre der Academie anständigen, bewohnbaren Zustand“. Da sein Brief kurzfristig keinerlei Resultate zeitigte, sah sich Klaproth wenige Monate später genötigt, ihm einen zweiten folgen zu lassen. Darin heißt es: „Ich muß nämlich anzeigen, dass ich keine einzige, zum akademischen Laboratorio gehörige Gerätschaft, kein Instrument oder Gefäß, kein chemisches Praeparat, überhaupt gar kein Inventarium-Stück, einen unbrauchbaren Blasebalg ausgenommen, gefunden habe." Als vorläufige Lösung schlug Klaproth vor, „es kann daher fürs erste mein eigener Apparat nothdürftig hinreichend sein“. Mit dem eigenen „Apparat" meinte er die Geräte und Instrumente aus der eigenen Apotheke, die er 1800 anlässlich seiner Ernennung zum Direktor des Akademielaboratoriums verkauft hatte. Klaproths wiederholte Beschwerden führten schließlich zum Neubau eines Laboratoriumshauses, das er im Dezember 1802 mitsamt der Ausstattung aus seinem Apothekerlaboratorium beziehen konnte. Die Grundausstattung dieses neuen, erst Anfang des 19. Jahrhunderts errichteten Laboratoriums der Preußischen Akademie der Wissenschaften stammte somit ebenfalls aus einem Apothekerlaboratorium.

\section{Diskussion}

Die Laboratorien der Frühen Neuzeit waren sowohl Räume des Wissens als auch der technologischen Innovation und gewerblichen Produktion. Sie waren handwerkliche Arbeitsstätten, in denen auch Naturforschung betrieben wurde, und speziell zur Naturforschung errichtete Räume und Häuser, in denen auch pharmazeutische, metallurgische und andere handwerkliche Herstellungstechniken sowie Praktiken der Identifikation von Materialien exploriert und in denen bis etwa Mitte des 18. Jahrhunderts chemische Medikamente auch direkt für den Verkauf produziert wurden. Die Öfen, Schmelztiegel, Retorten und anderen chemischen Geräte, mit denen diese Laboratorien ausgestattet waren, konnten gleichermaßen für die Produktion chemischer Medikamente, die Analyse von Mineralwässern und gefälschten Waren, die Kontrolle der Qualität von Farbstoffen oder die Ermittlung des Metallgehalts von Erzen verwendet werden wie für das Studium der Eigenschaften von Stoffen, die Analyse ihrer elementaren Zusammensetzung 
und die Erkundung ihrer chemischen Reaktionen. Dabei unterschied sich die materielle Kultur der handwerklichen Laboratorien, insbesondere die der Apothekerlaboratorien, bis ins frühe 19. Jahrhundert hinein nur graduell von der materiellen Kultur akademisch-chemischer Laboratorien. ${ }^{31}$ Von Mitte des 18. Jahrhunderts an wurden die akademischen (chemischen) Laboratorien zwar allmählich mit neuen "philosophischen“ Instrumenten ausgestattet, wie zum Beispiel mit Gefäßen für das Auffangen von Gasen, analytischen Feinwaagen, Thermometern, Barometern und Mikroskopen. An der Architektur und materiellen Grundausstattung der Laboratorien mit Öfen, Destillationsapparaturen und Instrumenten für Auflösungen, Filtrationen, Präzipitationen und Kristallisationen sowie für das Schmelzen und Kalzinieren von Stoffen änderte sich jedoch bis zur Jahrhundertwende nichts. Dasselbe gilt für die anderen großen Bereiche der materiellen Kultur, die Verfahrenstechniken, die maßgeblich an die vorhandenen Instrumenten gebunden waren, sowie die Rohmaterialien und chemischen Stoffe. Der Bestand chemisch reiner Reagenzien und Materialien in akademisch-chemischen Laboratorien erweiterte sich zwar im Verlauf des 18. Jahrhunderts, aber wenn alle Stoffe berücksichtigt werden, einschließlich der zahlreichen Pflanzen- und Tierstoffe, so ergeben sich auch hier große Übereinstimmungen zwischen der akademischen und der gewerblichen Welt (Klein/Lefèvre 2007). Die akademisch-chemischen Laboratorien des 18. Jahrhunderts und die alchemistischen Laboratorien in den Jahrhunderten davor waren tief verwurzelt in der Welt des Handwerks und Gewerbes. Die frühmodernen Laboratorien sind daher Institutionen, in denen neue Konstellationen von Wissenschaft, Technik und Gesellschaft und damit einer technoscience avant la lettre in den Brennpunkt historischer Forschung kommen.

In der insgesamt spärlichen Literatur über frühneuzeitliche Laboratorien sind diese meist als Orte des wissenschaftlichen Experimentierens aufgefasst worden. ${ }^{32}$ Die wenigen historischen Studien, die darüber hinaus auch die handwerklich-produktive Dimension frühneuzeitlicher Laboratorien beleuchten, haben das vorherrschende historische Bild von Laboratorien als ausschließlich wissenschaftliche Institutionen nur marginal zu korrigieren vermocht. „The appearance of the laboratory“, so der Chemiehistoriker Owen Hannaway, „is indicative of a new mode of scientific inquiry." (Hannaway 1986: 585, meine Hervorhebung) Auch der erst kürzlich erschienene Aufsatz des Chemiehistorikers Maurice Crosland hebt bereits im Titel („The Location of Experimental Science") hervor, unter welchen (unausgesprochenen) Voraussetzungen er frühmoderne Laboratorien diskutiert. Sein Resümee: „Perhaps the most basic feature of a laboratory is isolation from the everyday world; it must be far removed from the bustle, the jostling, the noise, the fluctuations of the market place." (Crosland 2005: 239) Nach Crosland zeichnet sich wissenschaftliches (chemisches) Experimentieren (wohl $\mathrm{zu}$ allen Zeiten) dadurch aus, dass es in deutlicher Abgrenzung und sogar 
„isoliert" von der alltäglichen Welt, zu der auch Handwerk und Märkte gehören, vonstatten geht.

Mit anderen Argumenten, im Resultat jedoch ähnlich, hat der Soziologe und Wissenschaftshistoriker Steven Shapin in seinem viel gepriesenen Essay The House of Experiment in Seventeenth-Century England (1988) die sozialen und epistemischen Grenzen der frühneuzeitlichen Laboratorien in England umrissen. Shapins Essay hat das heutige Bild frühneuzeitlicher Laboratorien in der Wissenschaftsgeschichte insgesamt wohl nachhaltiger geprägt als jede andere Studie zu diesem Gegenstand. Wie Crosland thematisiert auch Shapin die frühneuzeitlichen Laboratorien ausschließlich als Orte der „neuen experimentellen Wissenschaft“ im Singular (Shapin 1988: 377). Obwohl er in den einleitenden Abschnitten seines Essays zur Kenntnis nimmt, dass die Alchemisten der Frühen Neuzeit in Laboratorien experimentierten, übernimmt er das alte Klischee der Alchemie als einer Geheimwissenschaft schlechthin und grenzt sie damit aus seiner „neuen experimentellen Wissenschaft" aus. Denn für Letztere war nach Shapin Öffentlichkeit konstituierend, wobei er „Öffentlichkeit" im Hinblick auf Laboratorien vor allem als „öffentlichen Zugang“ zum Laboratoriumsraum und direkte Überprüfung experimenteller Fakten durch Augenzeugen definiert. Alchemistische und chemische Laboratorien sowie die mit ihnen eng verwandten Apothekerlaboratorien, die Shapin in der Einleitung seiner Arbeit durchaus erwähnt, geraten auf diese Weise im Hauptteil seines Essays aus dem Blickfeld. Denn Apothekerlaboratorien waren, ebenso wie metallurgische und andere handwerkliche Laboratorien, als private oder kommerzielle Werkstätten selbstverständlich nicht öffentlich zugänglich. ${ }^{33}$ Infolgedessen argumentiert Shapin, Laboratorien hätten als Orte „wissenschaftlichen“ Experimentierens im 17. Jahrhundert erst neu arrangiert und definiert werden müssen. Das neu zu definierende „wissenschaftliche“ Laboratorium musste demgemäß einerseits der Abgeschiedenheit eines Klosters gleichen, um dort ungestört experimentieren zu können, andererseits aber ein öffentlicher Raum sein, in dem experimentelle Fakten durch Augenzeugen bestätigt werden konnten. "Such a space did not then clearly exist“, so Shapin. „This space had necessarily to be carved out of and rearranged from existing domains of accepted public and private activity and existing stipulations about the proper uses of spaces." (Ebd.: 386) Wer jedoch meint, Shapin verstehe die bereits existierenden alchemistischen Laboratorien des 16. und 17. Jahrhunderts als Vorläufer der von ihm hervorgehobenen „wissenschaftlichen“ Laboratorien, sieht sich getäuscht. Das Modell für Robert Boyle und andere Mitglieder der Royal Society war nach Shapin vielmehr "the gentleman's private residence and, within it, its public room" (ebd.: 397). ${ }^{34}$

Shapin zieht somit eine scharfe Trennlinie, sowohl epistemisch als auch sozial, zwischen den frühneuzeitlichen akademischen Laboratorien, die vorwiegend der Naturforschung dienten, und den pharmazeutischen und 
anderen handwerklichen Laboratorien. Grundlage dieser Grenzziehung ist die zentrale epistemologische Funktion, die er dem räumlichen Zugang zum Laboratorium für die gelehrte Öffentlichkeit einräumt. Vergleichende Analysen der materiellen Kultur akademischer und handwerklicher Laboratorien kommen dagegen in seinem Essay ebenso wenig vor wie detaillierte Analysen der Laboratoriumspraktiken und ihrer Ziele. Vielmehr wird der öffentliche Zutritt zum Laboratoriumsraum zum Drehpunkt der gesamten historischen Analyse gemacht. Sollte es tatsächlich ein Hauptanliegen Boyles gewesen sein, Laboratorien neu zu definieren und seine eigene Experimentierstätte deutlich von den "dunklen und rußigen Laboratorien“ der Alchemisten abzugrenzen (ebd.: 378), so leuchtet nicht so recht ein, warum er den bei Alchemisten und Apothekern geläufigen Terminus „Laboratorium“ übernahm, anstatt eine neue, enger an die Terminologie der Experimentalphilosophie angelehnte Bezeichnung einzuführen wie „experimental cabinet", „experimental theater" oder „house of experiment" (Shapins Titel). Wie auch immer man diese Frage für Boyle und die Royal Society des 17. Jahrhunderts beantwortet, im 18. Jahrhundert und auf dem europäischen Kontinent war die Situation ohnehin anders. So gibt es keine Belege dafür, dass die Experimente im Laboratorium der Pariser Akademie der Wissenschaften und am Jardin Royal des Plantes prinzipiell und stets in Anwesenheit von Augenzeugen überprüft werden mussten. Ähnliches gilt für die Experimente Marggrafs, Achards und Klaproths an der Preußischen Akademie der Wissenschaften. Nach allem was wir wissen, hatten die drei Direktoren des Berliner Akademielaboratoriums im 18. und frühen 19. Jahrhundert nahezu exklusiven Zugang zu diesem Laboratorium, von den Laborjungen und Lieferanten abgesehen. ${ }^{35}$ War das Berliner Akademielaboratorium deshalb keine öffentliche wissenschaftliche Institution? Die Frage der wissenschaftlichen Öffentlichkeit entschied sich nicht an der Türschwelle zum Akademielaboratorium, sondern durch regelmäßige mündliche Berichte in den Sitzungen der Akademie, permanente Rechenschaftsablegung und Begründung von Anschaffungen gegenüber dem Direktorium und der ökonomischen Kommission der Akademie, öffentliche Vorlesungen mit Demonstrationsexperimenten und nicht zuletzt durch die zahlreichen schriftlichen Ver-Öffentlichungen.

So wichtig das Kriterium der Öffentlichkeit für die historische Analyse der neuzeitlichen Wissenschaften ist, so gibt es zweifellos weitere, nicht weniger relevante Gesichtspunkte. Im vorliegenden Aufsatz waren die „materielle Kultur" und der „materielle Transfer" von Instrumenten, Gefäßen und Materialen die zentralen Kategorien, die einen synoptischen Blick auf handwerkliche und akademische Laboratorien gestatteten, ohne damit Anspruch auf ein ausdifferenziertes Gesamtbild zu erheben. Es wurde gezeigt, dass das Laboratorium der Königlich Preußischen Akademie der Wissenschaften architektonisch und hinsichtlich seiner Ausstattung mit Instrumenten, Gefä- 
ßen und Materialien weitgehend mit den zeitgenössischen Apothekerlaboratorien übereinstimmte. Das house of experiment der Preußischen Akademie der Wissenschaften im 18. Jahrhundert war ein Ort der Naturforschung und zugleich ein "Arbeitshaus“, zwischenzeitlich sogar eine technologische Versuchsanstalt und „Zuckerfabrik“, das durch direkten materiellen Transfer von Instrumenten, Gefäßen und Materialien aus den Apothekerlaboratorien Marggrafs und Klaproths ausgestattet wurde. Es ist ein historischer Beleg für die enge Verflechtung frühneuzeitlicher akademischer und handwerklichgewerblicher Laboratorien.

\section{Anmerkungen}

1 „Frühe Neuzeit" bezieht hier das gesamte 18. Jahrhundert ein.

2 Siehe die ersten Belege für die Verwendung des Wortes laboratory im Oxford English Dictionary, die aus dem 17. Jahrhundert stammen und in denen durchweg von "Alchemie“ die Rede ist. Im Eintrag laboratory im Lexicon Technicum von John Harris wird „Laboratorium“ definiert als "a room fitted on purpose for Chymical Operations, and furnished with Variety of Furnaces and Instruments necessary to that Art" (Harris 1704-1710, Bd. 1, meine Hervorhebung). Diderots Encyclopédie gibt die folgende Definition für laboratoire: ein geschlossener Raum oder Teil eines Hauses, „der alle chemischen Utensilien (utensils) enthält, die man unter den Bezeichnungen Öfen, Gefäße und Instrumente zusammenfasst, und in denen chemische Operationen durchgeführt werden" (Diderot 1751-1780, 9: 145; meine Übersetzung). In Zedlers Universal-Lexikon von 1737 heißt es ebenfalls, unter einem „Laboratorium“ werde ein „zu chemischen Arbeiten“ nötiges Haus verstanden (Zedler 1732-1750, 16: 30).

3 Man könnte die frühneuzeitlichen Laboratorien als speziell eingerichtete Räume oder Häuser definieren, die für naturforschendes (chemisches) Experimentieren und innovatives handwerkliches Produzieren (und Kontrollieren) genutzt wurden. Methodisch halte ich mich in diesem Aufsatz jedoch strikt an den nachweisbaren Wortgebrauch von „Laboratorium".

4 Noch in der sechsten, neubearbeiteten Auflage von Meyers Konversations-Lexikon von 1909 ist „Laboratorium“ wie folgt definiert: „Das zur Ausführung chemischer Arbeiten bestimmte und mit den nötigen Vorrichtungen versehene Lokal."

5 Siehe Crosland 2005, Hannaway 1986, James 1989, Nye 1993, Shapin 1988, Smith 2000, 2006. James (1989: 3) bemerkt: „It is almost impossible to conceive of chemistry outside a laboratory environment; it was in this discipline that laboratories were first built and perfected as places where nature could be studied under controlled conditions." Hannaway (1986: 585) kommt zu folgendem Schluss: „Indications are that the laboratory was at first linked exclusively with alchemy and chemistry.“ Nye (1993: 50) stellt fest: „In the early nineteenth century, the meaning of ,laboratory' was irrefutably chemical." Shapin (1988: 377) bemerkt: „Among scores of English usages I have registered through the 1680s, I have not encountered one in which the space pointed to was without furnace, used as a nonportable source of heat for chemical or pharmaceutical operations.“

6 Ich verwende im Folgenden den Terminus „akademische Laboratorien“ als Ersatz für den anachronistischen Terminus „wissenschaftliche“ Laboratorien. Unter „akademischen Laboratorien“ sind demnach nicht nur diejenigen Laboratorien zu verstehen, die an Akademien angesiedelt waren, sondern alle Laboratorien, einschließlich der privaten, deren Schwerpunkt auf der experimentellen Naturforschung lag.

7 Siehe dazu Cook 2007, Klein/Spary in Vorbereitung, Roberts/Schaffer/Dear 2007, Smith 2006, Smith/Findlen 2002, Schiebinger/Swan 2005, Schabas/de Marchi 2003, Stewart 1992. 
8 Auf andere Bedingungen für diese Übergänge wie Ausbildung, Wissensformen, soziale Anreize und institutionelle Gelegenheiten bin ich andernorts ausführlich eingegangen (Klein 2007a).

9 Es liegt weiterhin nahe, die Kontinuität des explorativen wissenschaftlichen Experimentierens, wie wir sie etwa aus den „Experimentalsystemen“ (Rheinberger) des 20. Jahrhunderts kennen, mit der Einrichtung solcher privilegierter und spezialisierter Räume des Experimentierens zu verknüpfen.

10 „Technologisch“ ist hier in Anführungszeichen gesetzt, da das Wort „Technologie“ im deutschen Sprachraum erst vom späten 18. Jahrhundert an verwendet wurde. Die „Experimentalgeschichte" (experimental history) war ein experimenteller Stil, der in der Wissenschaftsgeschichte bisher weitgehend ignoriert wurde. Auch außerhalb der Chemie spielte die Experimentalgeschichte im 17. und 18. Jahrhundert eine große Rolle, unter anderem bei R. Hooke, C.-F. Dufay und R.-A. F. de Réaumur.

11 Homburg (1999: 6) gibt einen guten kursorischen Überblick über Universitätslaboratorien, pharmazeutische Laboratorien und chemische Fabriken im 18. Jahrhundert, der in folgender Feststellung mündet: „In the eighteenth century, there were only slight differences between university laboratories on the one hand and pharmaceutical laboratories and chemical factories on the other." Holmes (1989: 19f.) verstand dagegen die fortdauernden Übereinstimmungen zwischen den „wissenschaftlichen“ Laboratorien und den Laboratorien der Metallurgen, Probierer und Apotheker im 18. Jahrhundert als bloß „äußerliche“ - was immer dies genauer heißen mag - , da er die Chemie des 18. Jahrhunderts, im Unterschied zur Alchemie bis Mitte des 17. Jahrhunderts, als eine aus der handwerklichen Kunst herausgewachsene „experimentelle Wissenschaft" verstand.

12 Eine kurze Bemerkung über handwerkliche Produktion in alchemistischen Laboratorien befindet sich auch bei Newman/Principe (2002: 100). Dagegen gehen Priesner und Figala (1998: 208-215) sowie Schütt (2000) auf diesen Aspekt nicht ein.

13 In einem früheren Aufsatz hatte Smith (2000: 351) die Übereinstimmungen alchemistischer Laboratorien mit Werkstätten auf die Zeit vor dem 16. Jahrhundert begrenzt: „In the sixteenth century, the word laboratorium came to designate a specific site in which (usually chemical) experiments or trials were carried out. Before this time, ,laboratories' had been indistinguishable from apothecary shops and the workshops of artisans (indeed, laboratories continued frequently to be called officinae, or workshops).“

14 Die Ausstattung von Lavoisiers Laboratorium im Pariser Arsenal mit Präzisionsinstrumenten und speziell angefertigten pneumatisch-chemischen Apparaten war eine einzigartige Ausnahme.

15 Zum Laboratorium der Berliner Akademie der Wissenschaften siehe auch Dann 1958, Engel 1997.

16 Siehe „Preußisches Medizinaledikt“ von 1693 in Stürzbecher 1966: 43-64.

17 Siehe dazu insbesondere Schneider 1972, 1968-1974 sowie weiterhin Beisswanger 1968 und Krüger 1968. Ich halte mich hier strikt an die zeitgenössische Klassifikation und Bezeichnung "chemische Medikamente“.

18 Siehe dazu Klein 2007a und die dort zitierte Sekundärliteratur. Ein weiteres Beispiel für ein pharmazeutisches Laboratorium, an dem auch gelehrt wurde, ist das Laboratorium der Londoner Apothecary's Hall, siehe Simmons 2006.

19 Literaturhinweise und weitere Ausführungen über das Laboratorium der Berliner Hofapotheke befinden sich in Klein 2007a.

20 Macquer 1766 (erste Auflage), 1778 (zweite, verbesserte Auflage), 1789 (deutsche Übersetzung nach der zweiten Auflage). Zwischen der ersten Auflage gibt es interessante Unterschiede, die weiter unten angesprochen werden. Für die Übersetzung der Zitate der ersten Auflage übernehme ich weitgehend die deutsche Übersetzung der zweiten Auflage, ändere diese jedoch, wenn sie von der ersten Auflage abweicht.

21 Siehe das Zitat weiter oben.

22 Die hier von Macquer angesprochenen Unterschiede betrafen jedoch nicht alle Bereiche der handwerklichen Produktion, insbesondere nicht die Apothekerkunst. Abgesehen von den Destillationsapparaten stimmten die Instrumente des Apothekerlaboratoriums und des 
akademisch-chemischen Laboratoriums auch hinsichtlich ihrer Größenordnung überein. Größere Produktmengen wurden im Apothekergewerbe in der Regel durch Wiederholung der Operation oder mit einer größeren Anzahl von Gefäßen erzielt (mit Ausnahme der Destillation).

23 Diese Aussage bezieht sich auf die kollektive Ebene der Chemie. In Einzelfällen experimentierten Chemiker auch schon vor der Mitte des 18. Jahrhunderts gelegentlich mit philosophischen Instrumenten, siehe Holmes/Levere 2000.

24 In der deutschen Ausgabe wird cabinet de physique mit „Instrumentensammlungen der Naturforscher" übersetzt, siehe Macquer 1789, 4: 13.

25 Archiv der Berlin-Brandenburgischen Akademie der Wissenschaften, Bestand Preußische Akademie der Wissenschaften (1700-1811), I-XIII-19, Blätter 1-36 (im Folgenden abgekürzt als ABBAW), Harnack 1900, 1 (1): 487.

26 Um genau zu sein, es handelte sich um die Apotheke (beziehungsweise das Apothekerlaboratorium), die Marggrafs Vater gehörte.

27 Siehe Klein 2007a und die dort zitierte Sekundärliteratur.

28 ABBAW, I-XIII-20, Blätter 1-4. Das Inventarium wurde im Januar 1755 zusammengestelllt, siehe dazu auch Engel 1997.

29 Diese Liste trug den Titel „Ohngefehre Specification derer Instrumente und anderen zu einem Chymischen Laboratorio gehörigen Sachen, welche der Herr Director Marggraf selbst aus eigenen Mitteln angeschaffet nunmehr aber seiner Königlichen Academie zu adiren willens ist“. (ABBAW, I-XIII-20, Blätter 3, 4). Siehe auch Engel 1997.

30 Siehe insbesondere ABBAW, I-XVI-123, Blatt 27 (Abrechnungen für das Jahr 1757), ABBAW, I-XVI-128, Blatt 26 (für 1762), ABBAW, I-XVI-129, Blatt 26 (für 1763), ABBAW, I-XVI-135, Blatt 27 (für 1769).

$31 \mathrm{Zu}$ den Veränderungen im 19. Jahrhundert siehe Homburg 1999, Meinel 2000, Nawa/ Meinel 2007.

32 Siehe insbesondere Crosland 2005, Eklund 1975, Hannaway 1986, Holmes 1989, 2003, Shapin 1988, Shackelford 1993.

33 Dies bedeutet jedoch keineswegs, dass diese vorwiegend oder gar ausschließlich Produktionsstätten von kommerziellem oder alchemistischem Geheimwissen waren. Die Pharmakopöen und andere Apothekerbücher der Zeit sprechen eine andere Sprache. Auf das komplexe Verhältnis zwischen dem aus kommerziellen Gründen geheim gehaltenen handwerklichen Wissen und dem öffentlichen Wissen kann hier nicht eingegangen werden, siehe dazu Long 2001.

34. Shapin stützt diese These hauptsächlich auf Äußerungen Robert Boyles, seine Evidenz ist jedoch sehr fragmentarisch.

35 Gelegentliche Besuche in Laboratorien waren jedoch überall in Europa üblich, darüber liegen für das Preußische Akademielaboratorium auch einzelne Berichte vor.

\section{Literatur}

ABBAW, I-XIII-20, Blätter 9-16.

ABBAW, I-XIII-20, Blatt 7.

ABBAW, I-XVI-134, Blätter 26, 29.

ABBAW, I-XIII-20, Blätter 9-16.

ABBAW, I-XIII-20, Blatt 14.

ABBAW, I-XIII-20, Blätter 10, 11.

ABBAW, I-XIII-26, Blatt 1.

ABBAW, I-XIII-26, Blatt 11.

ABBAW, I-XIII-26: Blätter 65, 66.

ABBAW, I-XIII-26: Blatt 131. 
Archiv der Berlin-Brandenburgischen Akademie der Wissenschaften (ABBAW), Bestand PreuBische Akademie der Wissenschaften (1700-1811).

Beisswanger, Gabriele, 1968. Arzneimittelversorgung im 18. Jahrhundert. Die Stadt Braunschweig und die ländlichen Distrikte im Herzogtum Braunschweig-Wolfenbüttel. Braunschweig: Deutscher Apothekerverlag.

Cook, Harold J., 2007. Matters of Exchange. Commerce, Medicine, and Science in the Dutch Golden Age. New Haven/London: Yale University Press.

Cooper, Michael, 2003. Hooke's Career. In: Jim Bennett, Michael Cooper, Michael Hunter und Lisa Jardine, Hg., London's Leonardo. The Life and Work of Robert Hooke. Oxford: Oxford University Press, 1-62.

Crosland, Maurice P., 2005. Early Laboratories c. 1600-c.1800 and the Location of Experimental Science. Annals of Science, 62, 233-253.

Dann, Georg Edmund, 1958. Martin Heinrich Klaproth (1743-1817). Ein deutscher Apotheker und Chemiker, sein Weg und seine Leistung. Berlin: Akademie-Verlag.

Diderot, Denis J./LeRond d'Alembert, Jean, 1966. Encyclopédie ou dictionnaire raisonné des sciences, des arts, et des métiers. 35 Bde. Stuttgart: Frommann [Nachdruck der ersten Edition Paris 1751-1780].

Dilg, Peter, 2000. Die Apotheke als Forschungsstätte. Berichte zur Wissenschaftsgeschichte, 23, 303-315.

Eklund, John, 1975. The Incompleat Chymist. Being an Essay on the Eighteenth-Century Chemist in his Laboratory, with a Dictionary of Obsolet Chemical Terms of the Period. Washington: Smithonian Institution Press.

Engel, Britta, 1997. Das Berliner Akademielaboratorium zur Zeit Marggrafs und Achards. Mitteilungen der Fachgruppe „Geschichte der Chemie“ in der Gesellschaft Deutscher Chemiker, $13,3-12$.

Hagen, Karl Gottfried, 1786. Lehrbuch der Apothekerkunst. Königsberg und Leipzig: Gottfried L. Hartung [3. Aufl.].

Hannaway, Owen, 1986. Laboratory Design and the Aim of Science. Andreas Libavius versus Tycho Brahe. Isis, 77, 585-610.

Harnack, Adolf. 1900. Geschichte der Königlich Preussischen Akademie der Wissenschaften zu Berlin, 3 Bde. Berlin: Reichsdruckerei.

Harris, John, 1704-1710. Lexicon Technicum. Or an Universal English Dictionary of Arts and Sciences, Explaining not only the Terms of Art, but also the Arts Themselves. London: Dan. Brown et al.

Holmes, Frederic Lawrence, 1989. Eighteenth-Century Chemistry as an Investigative Enterprise. Berkeley: University of California Press.

Holmes, Frederic L., 2003. Laboratory, Chemical. In: John L. Heilbron u.a., Hg., The Oxford Companion to the History of Modern Science. Oxford: Oxford University Press, 441-442.

Holmes, Frederic L./Levere, Trevor H., Hg., 2000. Instruments and Experiments in the History of Chemistry. Cambridge, Mass./ London: The MIT Press.

Homburg, Ernst, 1999. The Rise of Analytical Chemistry and its Consequences for the Development of the German Chemical Profession (1780-1860). Ambix, 46, 1-31.

Hörmann, Johannes, 1898. Die Königliche Hofapotheke in Berlin 1598-1898. HohenzollernJahrbuch. Forschungen und Abbildungen zur Geschichte der Hohenzollern in BrandenburgPreußen, 2, 208-226.

James, Frank A. J. L., 1989. Introduction. In: James, Frank A. J. L., Hg., The Development of the Laboratory. Essays on the Place of Experiment in Industrial Civilisation. New York: American Institute of Physics, 1-7.

Klein, Ursula, 2005. Technoscience avant la lettre. Perspectives on Science, 13, 227-66.

Klein, Ursula, 2007a. Apothecary-Chemists in Eighteenth-Century Germany. In: Lawrence M. Principe, Hg., New Narratives in Eighteenth Century Chemistry. Dordrecht: Springer, 97-137.

Klein, Ursula, 2007b. Apothecary's Shops, Laboratories and Chemical Manufacture in Eighteenth-Century Germany. In: Lissa Roberts, Simon Schaffer und Peter Dear, Hg., The Mind- 
ful Hand. Inquiry and Invention from the Late Renaissance to Early Industrialisation. Amsterdam: Royal Netherlands Academy of Arts and Sciences, 247-276.

Klein, Ursula, in Vorbereitung. Blending Technical Innovation and Learned Natural Knowledge. The Making of Ethers. In: Ursula Klein und Emma Spary, Hg., Between Marketplace and Laboratory. Materials and Expertise in Early Modern Europe, 1500-1800.

Klein, Ursula/Lefèvre, Wolfgang, 2007. Materials in Eighteenth-Century Science. A Historical Ontology. Cambridge, Mass./London: The MIT Press.

Klein, Ursula/Spary, Emma, in Vorbereitung. Between Laboratory and Market. Materials and Expertise in Early Modern Europe, 1500-1800.

Köhnke, Otto, 1900. Gesammtregister über die in den Schriften der Akademie von 1700-1899 erschienenen wissenschaftlichen Abhandlungen und Festreden. Berlin: Reichsdruckerei.

Krüger, Mechthild, 1968. Zur Geschichte der Elixiere, Essenzen und Tinkturen. Braunschweig: Technische Hochschule.

Krünitz, Johann Georg, 1773-1858. Oeconomische Encyclopädie, oder allgemeines System der Land-, Haus- und Staats-Wirthschaft in alphabetischer Ordnung. 242 Teile. Berlin: J. Pauli und E. Litfaß.

Livingstone, David N., 2003. Putting Science in its Place. Geographies of Scientific Knowledge. Chicago: University of Chicago Press.

Long, Pamela O., 2001. Openness, Secrecy, Authorship. Technical Arts and the Culture of Knowledge from Antiquity to the Renaissance. Baltimore: Johns Hopkins University Press.

Macquer, Pierre Joseph, 1766. Dictionnaire de chimie, contenant la théorie et la pratique de cette science, son application à la physique, à l'histoire naturelle, à la médicine et à l'Economie animale. Paris: Lacombe.

Macquer, Pierre Joseph, 1778. Dictionnaire de chimie, contenant la théorie et la pratique de cette science, son application à la physique, à l'histoire naturelle, à la médicine et aux arts dépendans de la chimie. 2 Bde. Paris: L'Imprimerie de Monsieur [2. Aufl.].

Macquer, Peter Joseph, 1789. Chymisches Wörterbuch oder Allgemeine Begriffe der Chymie nach alphabetischer Anordnung, 7 Bde. Leipzig: Weidmann.

Mauskopf, Seymour H., 2006. Pellets, Pebbles and Prisms: British Munitions for Larger Guns, 1860 - 1885. In: Brenda J. Buchanan, Hg., Gunpowder, Explosives and the State. A Technological History. Aldershot: Ashgate, 202-229.

Meinel, Christoph, 2000. Chemische Laboratorien. Funktion und Disposition. Berichte zur Wissenschaftsgeschichte, 23, 287-302.

Meynell, Guy, 1994. The Académie des Sciences at the Rue Vivienne, 1666-1699. Archives Internationale D'Histoire des Sciences, 44, 22-37.

Moran, Bruce T., 1991. Chemical Pharmacy Enters the University. Johannes Hartmann and the Didactic Care of Chymiatria in Early Seventeenth Century. Madison: American Institute of Pharmacy.

Müller, Hans-Heinrich, 2002. Franz Carl Achard, 1753-1821, Biographie. Berlin: Dr. Albert Bartens.

Nawa, Christine/Meinel, Christoph, 2007. Instrumente und Apparaturen in Heidelberger Laboratorien skizziert von Friedrich Veith (1817-1907). Regensburg: HC-Druck, Kneiting.

Newman, William R./Principe, Lawrence M., 2002. Alchemy Tried in the Fire. Starkey, Boyle, and the Fate of Helmontian Chymistry. Chicago: The University of Chicago Press.

Nieto-Galan, Agustí, 2001. Colouring Textiles. A History of Natural Dyestuffs and Industrial Europe. Dordrecht: Kluwer.

Nye, Mary Jo, 1993. From Chemical Philosophy to Theoretical Chemistry. Dynamics of Matter and Dynamics of Disciplines, 1800-1950. Berkeley: University of California Press.

Pilcher, Richard B., 1938. Boyle's Laboratory. Ambix, 2, 17-24.

Priesner, Claus/Figala, Karin, 1998. Alchemie. Lexikon einer hermetischen Wissenschaft. München: C. H. Beck.

Puschner, Johann G., um 1720. Amoenitates Altdorfinae oder eigentliche nach dem Leben gezeichnete Prospecten der löblichen Universität Altdorf [...]. Nürnberg: Michahelles.

Roberts, Lissa/Schaffer, Simon/Dear, Peter, Hg., 2007. The Mindful Hand. Inquiry and Inven- 
tion from the Late Renaissance to Early Industrialisation. Amsterdam: Royal Netherlands Academy of Arts.

Sargent, Rose-Mary, 1995. The Different Naturalist. Robert Boyle and the Philosophy of Experiment. Chicago: University of Chicago Press.

Schabas, Margaret/ De Marchi, Neil, Hg., 2003. Oeconomics in the Age of Newton. Durham: Durham University Press.

Schiebinger, Londa/Swan, Claudia, Hg., 2005. Colonial Botany. Science, Commerce, and Politics in the Early Modern World. Philadelphia: University of Pennsylvania Press.

Schneider, Wolfgang, 1968-74. Lexikon zur Arzneimittelgeschichte. Sachwörterbuch zur Geschichte der pharmazeutischen Botanik, Chemie, Mineralogie, Pharmakologie, Zoologie. 7 Bde. Frankfurt a. M.: Govi-Verlag.

Schneider, Wolfgang, 1972. Geschichte der pharmazeutischen Chemie. Weinheim: Verlag Chemie.

Schütt, Hans-Werner, 2000. Auf der Suche nach dem Stein der Weisen. Die Geschichte der Alchemie. München: C. H. Beck.

Shackelford, Jole, 1993. Tycho Brahe, Laboratory Design, and the Aim of Science. Reading Plans in Context. Isis, 84, 211-230.

Shapin, Steven, 1988. The House of Experiment in Seventeenth-Century England. Isis, 79, 373403.

Shapin, Steven, 1989. Who Was Robert Hooke. In: Michael Hunter und Simon Schaffer, Hg., Robert Hooke. New Studies. Woodbridge: The Boydell Press, 253-286.

Simcock, A. V., 1984. The Ashmolean Museum and Oxford Science 1683-1983. Oxford: Museum of the History of Science.

Simmons, Anna, 2006. Medicines, Monopolies and Mortars. The Chemical Laboratory and Pharmaceutical Trade at the Society of Apothecaries in the Eighteenth Century. Ambix, 53, 221-236.

Simpson, A. D. C., 1989. Robert Hooke and Practical Optics. Technical Support at a Scientific Frontier. In: Michael Hunter und Simon Schaffer, Hg., Robert Hook. New Studies. Woodbridge: The Boydell Press, 33-61.

Smith, Pamela, 1994. The Business of Alchemy. Science and Culture in the Holy Roman Empire. Princeton: Princeton University Press.

Smith, Pamela, 2000. Laboratories. In: Wilbur Applebaum, Hg., Encyclopedia of the Scientific Revolution. From Copernicus to Newton. New York: Garland, 351-353.

Smith, Pamela, 2006. Laboratories. In: Katherine Park und Lorraine Daston, Hg., The Cambridge History of Science. Band 3: Early Modern Science. Cambridge: Cambridge University Press, 290-305.

Smith, Pamela H./Findlen, Paula, Hg., 2002. Merchants and Marvels. Commerce, Science and Art in Early Modern Europe. New York: Routledge.

Soukup, Rudolf Werner, 2007. Chemie in Österreich (Bergbau, Alchemie und frühe Chemie). Von den Anfängen bis zum Ende des 18. Jahrhunderts. Wien/Köln/Weimar: Böhlau.

Soukup, Rudolf Werner/Mayer, Helmut, 1997. Alchemistisches Gold, Paracelsische Pharmaka. Laboratoriumstechnik im 16. Jahrhundert. Chemiegeschichtliche and archäometrische Untersuchungen am Inventar des Laboratoriums von Oberstockstall/Kirchberg am Wagen. Wien/Köln/Weimar: Böhlau.

Stewart, Larry, 1992. The Rise of Public Science. Rhetoric, Technology and Natural Philosophy in Newtonian Britain, 1660-1750. Cambridge: Cambridge University Press.

Stroup, Alice, 1990. A Company of Scientists. Botany, Patronage, and Community at the Seventeenth-Century Parisian Royal Academy of Sciences. Berkeley: University of California Press.

Stürzbecher, Manfred, 1966. Beiträge zur Berliner Medizingeschichte. Quellen und Studien zur Geschichte des Gesundheitswesens vom 17. bis zum 19. Jahrhundert. Berlin: Walter de Gruyter.

Turner, Antony, 1987. Early Scientific Instruments. Europe 1400-1800. London: Sotheby's Publication.

Weyer, Jost, 1992. Graf Wolfgang II. von Hohenlohe und die Alchemie. Alchemistische Studien im Schloß Weikersheim, 1587-1610. Sigmaringen: J. Thorbeke. 
Wiesenfeldt, Gerhard, 2002. Leerer Raum in Minervas Haus. Experimentelle Naturlehre an der Universität Leiden, 1675-1715. Amsterdam: Koninklijke Nederlandse Akademie van Wetenschappen.

Winter, Eduard, 1957. Die Registres der Berliner Akademie der Wissenschaften 1746-1766. Dokumente für das Wirken Leonhard Eulers in Berlin. Berlin: Akademie-Verlag.

Zedler, Johann Heinrich, 1732-1750. Grosses vollständiges Universal Lexicon aller Wissenschafften und Künste. 64 Bände. Halle/Leipzig: J. H. Zedler.

\section{Ursula Klein}

Max-Planck-Institut für Wissenschaftsgeschichte

Boltzmannstraße 22

D-14195 Berlin

E-Mail: klein@mpiwg-berlin.mpg.de 